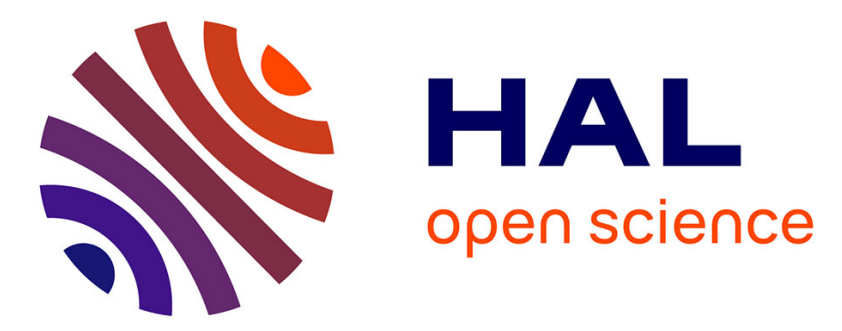

\title{
Random projection depth for multivariate mathematical morphology
}

Santiago Velasco-Forero, Jesus Angulo

\section{To cite this version:}

Santiago Velasco-Forero, Jesus Angulo. Random projection depth for multivariate mathematical morphology. IEEE Journal of Selected Topics in Signal Processing, 2012, 6 (7), pp.753-763. 10.1109/JSTSP.2012.2211336 . hal-00751347

\section{HAL Id: hal-00751347}

https://hal-mines-paristech.archives-ouvertes.fr/hal-00751347

Submitted on 13 Nov 2012

HAL is a multi-disciplinary open access archive for the deposit and dissemination of scientific research documents, whether they are published or not. The documents may come from teaching and research institutions in France or abroad, or from public or private research centers.
L'archive ouverte pluridisciplinaire HAL, est destinée au dépôt et à la diffusion de documents scientifiques de niveau recherche, publiés ou non, émanant des établissements d'enseignement et de recherche français ou étrangers, des laboratoires publics ou privés. 


\title{
Random Projection Depth for Multivariate Mathematical Morphology
}

\author{
Santiago Velasco-Forero, Student Member, IEEE, and Jesús Angulo
}

\begin{abstract}
The open problem of the generalization of mathematical morphology to vector images is handled in this paper using the paradigm of depth functions. Statistical depth functions provide from the "deepest" point a "center-outward ordering" of a multidimensional data distribution and they can be therefore used to construct morphological operators. The fundamental assumption of this data-driven approach is the existence of "background/foreground" image representation. Examples in real color and hyperspectral images illustrate the results.
\end{abstract}

Index Terms-Hyperspectral images, multivariate morphology, statistical depth function.

\section{INTRODUCTION}

M ATHEMATICAL morphology (MM) operators in modern image analysis are a set of powerful, robust and computationally efficient tools with multiple applications including image filtering, segmentation and visualization [2]. $\mathrm{MM}$ is a nonlinear image processing methodology based on the application of lattice theory to spatial structures [3]. It requires the definition of a complete lattice structure, i.e., an ordering among the pixels to be analyzed. However, there is not difficult to see that the idea of order is entirely absent from multivariate scene, i.e., there is no unambiguous means of defining the minimum and maximum values between two vectors of more than one dimension. Accordingly, the extension of MM to vector spaces, for instance, color/multi/hyper/ultraspectral images, is neither direct nor trivial because the pixels in the images are vectors. We refer keen readers to [4], [5] for a comprehensive review of vector morphology. In his seminal paper about multivariate ordering, Barnet [6] identified four families of ordering for vectors.

- The marginal ordering (M-ordering), is a trivial approach consisting in applying grayscale morphology techniques to each channel separately, that has been called marginal morphology in the literature [7]. However, the marginal approach is often unacceptable in several applications

Manuscript received February 03, 2012; revised May 03, 2012 and July 17, 2012; accepted July 24, 2012. Date of publication nulldate; date of current version October 12, 2012. This is an extended version of a paper that appeared at the 10th International Symposium of Mathematical Morphology and Its Applications to Image and Signal Processing held July 6-8 in Vergania-Intra, Italy [1]. The associate editor coordinating the review of this manuscript and approving it for publication was XXXXX XXXX.

The authors are with the Centre for Mathematical Morphology, MINES ParisTech, 77305 Fontainebleau Cedex, France (e-mail: velasco@cmm.ensmp.fr).

Color versions of one or more of the figures in this paper are available online at http://ieeexplore.ieee.org.

Digital Object Identifier 10.1109/JSTSP.2012.2211336 because, when morphological techniques are applied independently to each image channel, analysis techniques are subject to the well-known problem of false colors [8]; that is, it is very likely that new spectral constituents (not present in the original image) may be created as a result of processing the channels separately.

- To strictly preserve input vectors, the conditional ordering (C-ordering) approach, also known as lexicographic ordering, is frequently used. The C-ordering is based on the ordering of the components selected sequentially according to different conditions or priorities. When all the components are used, the $\mathrm{C}$-ordering is a total ordering [9].

- The reduced ordering (R-ordering) which performs the ordering of vectors in some scalar space, computed from a mapping of the vector onto a different representation where the ordering is naturally defined, typically distances or projections onto a dimensionality reduced space (using for instance the principal component analysis). For instance, Mahalanobis distance has been employed in several works on multivariate morphology including the information from a reference set [10]. Recently, reduced supervised ordering has shown be useful in the analysis of high dimensional images [11], [12].

- The P-ordering, is based on the partition of the vectors into groups, such that the groups can be distinguished with respect to rank or extremeness. Recently, approaches using combinatorial techniques and median/anti-median filters have been also used to construct ordering [13], [14]. There is however a problem of these latter approaches: the ordering is locally depending on the values of the spatial window, consequently it is not a partial ordering for the set of vectors in an image, i.e., dilation (erosion) obtained does not commute with the supremum (infimum) and the distributive property is not valid.

In this paper, a P-ordering for pixels in multivariate images is presented. To the best of our knowledge, this is the first approach which uses P-ordering to extend MM to multivariate image. Fig. 1 gives the intuition behind our approach. The paper is organized as follows. Restricting ourselves to data in vector spaces, in Section II the statistical depth functions definition is reviewed. This is the basic ingredient for the construction of P-ordering. The case of projection depth function is analyzed in detail and its convergence to classical Mahalanobis distance is presented for elliptically contoured distributions. Section III analyzes the application of ordering based on projected depth function in the context of vector images and it presents some interesting properties for practical problems in image processing. 


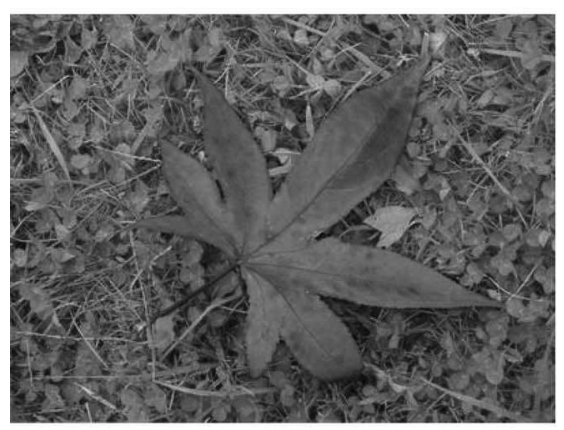

(a)

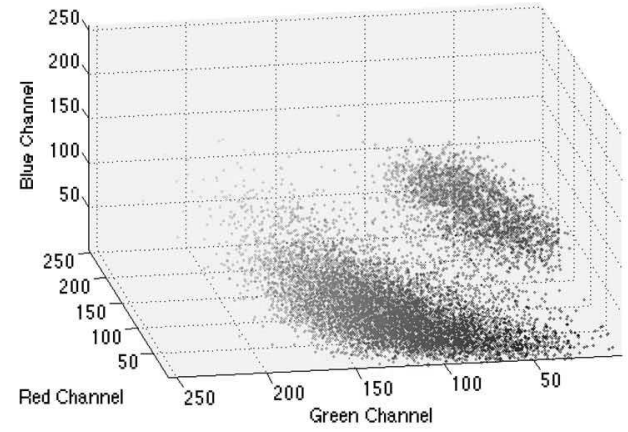

(b)

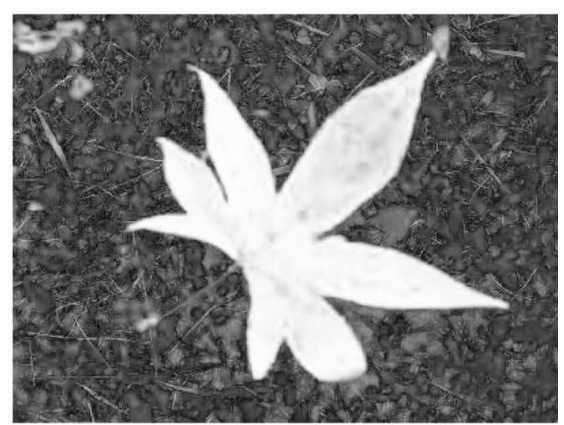

(c)

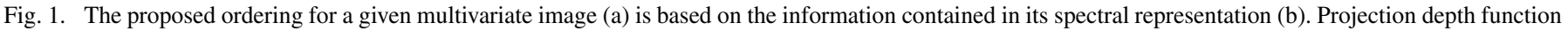

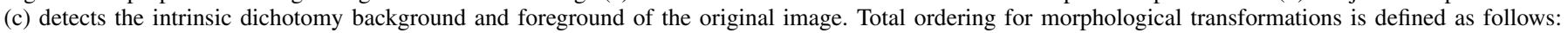
$\mathbf{x}_{1}<\mathbf{x}_{2} \Leftrightarrow P D\left(\mathbf{x}_{1} ; \mathbf{I}\right)<P D\left(\mathbf{x}_{2} ; \mathbf{I}\right)$.

Section IV shows the effectiveness of the proposed approach via practical examples and visual comparison in image enhancement, simplification and segmentation. Finally, Section V concludes the paper.

\section{STATISTICAL DEPTH FUnCTIONS}

\section{A. Notation}

Let us make precise the terms and notation to be used in the rest of the paper. Let $\mathrm{E}$ be a subset of the discrete space $\mathbb{Z}^{2}$, considered as the support space of the $2 \mathrm{D}$ image, and $\mathbb{F} \subseteq \mathbb{R}^{d}$ be a set of pixels values in dimension $d$, corresponding to the space of values of the multivariate image with $d$ channels. A vector-value image is represented by the mapping,

$$
\mathbf{I}:\left\{\begin{array}{lll}
\mathrm{E} & \rightarrow & \mathbb{F} \\
x=(i, j) & \rightarrow & \mathbf{x}
\end{array}\right.
$$

i.e., $\mathbf{I} \in \mathcal{F}(\mathrm{E}, \mathbb{F})$ the set of maps from a point $x$ at the discrete spatial coordinates $(i, j) \in \mathrm{E}$ into a vector value $\mathbf{x} \in \mathbb{F} \subseteq \mathbb{R}^{d}$. Let us assume that the pixel $x$ is represented by a $d$-dimensional vector $\mathbf{x}(i, j)=\left[x_{1}(i, j), x_{2}(i, j), \ldots, x_{d}(i, j)\right] \in \mathbb{R}^{d}$, where $\mathbb{R}$ denotes the set of real numbers in which the pixel's spectral response $x_{l}(i, j)$ at sensor channels $l=1, \ldots, d$. Additionally, let the data matrix $\mathbf{X}_{\mathbf{I}}$ be an $n \times d$ matrix representing $d$ spectral bands for each $n$ pixels in the vector-value image $\mathbf{I}$. In fact, $\mathbf{X}_{\mathbf{I}}$ is a matrix, with a slight abuse of notation we denote it as $\mathbf{X}$ for make easier the presentation.

\section{B. Statistical Depth Function}

Depth functions for multivariate data have been pursued in nonparametric data analysis and robust inference [15]. Depth functions assign to each point its degree of centrality with respect to a data cloud or a probability distribution. A depth function suitable for a distribution $\mathfrak{F}$ in $\mathbb{R}^{d}$, denoted by $D(\mathbf{x} ; \mathfrak{F})$, brings out the non-central ranking of the vector $\mathbf{x}$ in $\mathbb{R}^{d}$ with respect to $\mathfrak{F}$. A number of depth functions are available in the literature, for instance halfspace depth [16], simplicial depth [17], projection depth [18], spatial depth [19], Mahalanobis depth [15], etc. Roughly speaking, for a distribution $\mathfrak{F} \in \mathbb{R}^{d}$, a corresponding depth function $D(\mathbf{x} ; \mathfrak{F})$ provides an $\mathfrak{F}$-based center- outward ordering of point $\mathbf{x} \in \mathbb{R}^{d}$. Hence, $D(\mathbf{x} ; \mathfrak{F})$ is a function $\mathbb{R}^{d} \rightarrow \mathbb{R}$. Depth-based methods are completely data-driven and avoid strong distributional assumption. Moreover, they provide intuitive visualization of the data set via depth contours for a low dimensional input space. Analogous to linear order in one dimension, statistical depth functions provide an ordering of all points from the center outward in a multivariate data set, where the median is the "deepest" point in the data set. This leads to center-outward ordering to points and to a description in terms of nested contours. Let us start by a formal definition of a depth function.

Definition 1: [15], [17] A statistical depth function is a bounded nonnegative mapping $D(\cdot ; \cdot): \mathbb{R}^{d} \times \mathfrak{F} \rightarrow \mathbb{R}$ satisfying

1) $D\left(\mathbf{A x}+\mathbf{b} ; \mathfrak{F}_{\mathbf{A x}+\mathbf{b}}\right)=D(\mathbf{x} ; \mathfrak{F})$ holds for any random vector $\mathbf{x}$ in $\mathbb{R}^{d}$, any $d \times d$ nonsingular matrix $\mathbf{A}$, and any $\mathbf{b} \in \mathbb{R}^{d}$. That invariance to affine transformation means, the depth of a vector $\mathbf{x} \in \mathbb{R}^{d}$ should not depend on the underlying coordinate system or, in particular, on the scales of the underlying measurements.

2) $D(\boldsymbol{\theta} ; \mathfrak{F})=\sup _{\mathbf{x} \in \mathbb{R}^{d}} D(\mathbf{x} ; \mathfrak{F})$ holds for any $\mathfrak{F}$ having center $\boldsymbol{\theta}$. That means, for any distribution having a unique "center," the depth function should attain maximum value at this center.

3) $D(\mathbf{x} ; \mathfrak{F}) \leq D(\theta+\alpha(\mathbf{x}-\theta)$; $\mathfrak{F})$ holds for any $\mathfrak{F}$ having a deepest point $\theta$ and any $\alpha \in[0,1]$, i.e., as a point $\mathbf{x} \in \mathbb{R}^{d}$ moves away from the "deepest point" along any fixed ray through the center, the depth at $\mathbf{x}$ should decrease monotonically.

4) $D(\mathbf{x} ; \mathfrak{F}) \rightarrow 0$ as $\|\mathbf{x}\| \rightarrow \infty$, for each $\mathfrak{F}$, i.e., the depth of a point $\mathbf{x}$ should approach to zero as its norm approaches infinity.

In the sequel, we focus on the empirical version of the projection depth function [18], and we describe some useful properties to support it as a convenient option to produce P-ordering in vector spaces. Other statistical depth functions have considered in our preliminary work [1] for vector morphology. However, (random) projection depth function presents the best trade-off between robustness and computation time. We notice that the sense of the depth function is inverted, from the "unique center point" to outliers, in order to be compatible with mathematical morphology processing as introduced in Section III. 


\section{Projection Depth Function}

The basic concept of a projection depth function was introduced by D. Donoho [18] and posteriorly developed by Y. Zuo [15], [20]. It defines the measures of centrality for a vector $\mathbf{x}$ with respect to a multivariate distribution or a multivariate data cloud $(\mathbf{X})$ as the worst case outlyingness with respect to the one-dimensional scale functional in any one-dimensional projection, that is,

Definition 2: [18] The projection depth function for a vector $\mathbf{x}$ according with a data cloud $\mathbf{X}=\left[\mathbf{x}_{1}, \ldots, \mathbf{x}_{n}\right]$ as follows,

$$
P D(\mathbf{x} ; \mathbf{X})=\sup _{\mathbf{u} \in \mathbb{S}^{d-1}} \frac{\left|\mathbf{u}^{T} \mathbf{x}-M E D\left(\mathbf{u}^{T} \mathbf{X}\right)\right|}{M A D\left(\mathbf{u}^{T} \mathbf{X}\right)}
$$

where MED is the median and MAD is the median absolute deviation (MAD) and $\mathbb{S}^{d-1}=\left\{\mathbf{x} \in \mathbb{R}^{d}:\|\mathbf{x}\|_{2}=1\right\}$ is the $d$-dimensional hypersphere. MAD is a robust estimator of variability attributed to Gauss in 1816 [21]. The pair of robust estimators (MED,MAD) is included in (2) because they are not unduly affected by outliers [20]. Projection depth has been used to robust multivariate classification [22] and classification of functional data [23]. Note that $P D(\mathbf{x} ; \mathbf{X})$ is impossible to calculate in practice, because it requires the analysis for an infinite set of random projections. Our approach follows the suggestion of [24]: replacing the supremum in (2) by a maximum over a finite number of randomly chosen projection, obtaining a stochastic approximation of the random projection depth. The same argument have been used in [25] for other type of statistical depth function. Thus, we can calculate an approximate value of $P D(\mathbf{x} ; \mathbf{X})$ by using $k$ random projections uniformly distributed in $\mathbb{S}^{d-1}$ as follow

$$
P D(\mathbf{x} ; k, \mathbf{X})=\max _{\mathbf{u} \in \mathcal{U}} \frac{\left|\mathbf{u}^{T} \mathbf{x}-\operatorname{MED}\left(\mathbf{u}^{T} \mathbf{X}\right)\right|}{\operatorname{MAD}\left(\mathbf{u}^{T} \mathbf{X}\right)}
$$

where $\mathbb{U}=\left\{\mathbf{u}_{1}, \mathbf{u}_{2}, \ldots, \mathbf{u}_{k}\right\}$ with $\mathbf{u}_{i} \in \mathbb{S}^{d-1}$. Clearly, if $k \rightarrow$ $\infty$ then $P D(\mathbf{x} ; k, \mathbf{X}) \rightarrow P D(\mathbf{x} ; \mathbf{X})$.

\section{Equivalence in Elliptically Symmetric Distribution}

The depth function admits an analytical formulation when elliptically symmetric random variables are considered.

Definition 3: [26] A $d \times 1$ random vector $\mathbf{x}$ is said to have an elliptically symmetric distribution with parameters $\boldsymbol{\mu}_{(d \times 1)}$ and $\Sigma_{(d \times d)}$ if

$$
\mathbf{x} \stackrel{\text { dist }}{=} \boldsymbol{\mu}+\mathbf{A}^{T} \mathbf{y}
$$

where, $\mathbf{A}^{T} \mathbf{A}=\boldsymbol{\Sigma}$ with $\operatorname{rank}(\boldsymbol{\Sigma})=r$. Where $\mathbf{y} \stackrel{\text { dist }}{=} \alpha \mathbf{u}^{(r)}$, $\alpha \in \mathbb{R}, \mathbf{u}^{(r)}$ denote a random vector distributed uniformly on the unit sphere surface in $\mathbb{R}^{r}$ and $\stackrel{\text { dist }}{=}$ means equality in distribution.

Proposition 1: [26] The $d$-dimension random vector $\mathbf{X}$ has a multivariate elliptical distribution, written as $\mathbf{X} \sim E_{d}(\boldsymbol{\mu}, \boldsymbol{\Sigma}, \psi)$, if its characteristic function can be expressed as:

$$
\phi_{\mathbf{X}}=\exp \left(i \mathbf{t}^{T} \boldsymbol{\mu}\right) \psi\left(\frac{1}{2} \mathbf{t}^{T} \mathbf{\Sigma} \mathbf{t}\right)
$$

for some vector $\boldsymbol{\mu}$, positive-definite matrix $\boldsymbol{\Sigma}$, and for some function $\psi$, which is called the characteristic generator.
From $\mathbf{X} \sim E_{d}(\boldsymbol{\mu}, \boldsymbol{\Sigma}, \psi)$, it does not generally follow that $\mathbf{X}$ has a density $f_{\mathbf{X}}(\mathbf{x})$, but, if it exists, it has the following form:

$$
f_{\mathbf{X}}(\mathbf{x})=\frac{c_{d}}{\sqrt{|\Sigma|}} g_{d}\left[\frac{1}{2}(\mathbf{x}-\boldsymbol{\mu})^{T} \boldsymbol{\Sigma}^{-1}(\mathbf{x}-\boldsymbol{\mu})\right]
$$

where $c_{d}$ is the normalization constant and $g_{d}$ is some nonnegative function with $((d / 2)-1)$-moment finite. $g_{d}$ is called density generator [26]. In this case we shall use the notation $E_{d}\left(\boldsymbol{\mu}, \boldsymbol{\Sigma}, g_{d}\right)$ instead of $E_{d}(\boldsymbol{\mu}, \boldsymbol{\Sigma}, \psi)$.

Proposition 2: If $\mathrm{x}$ has a symmetric probability density function $\Phi(\cdot)$ that is continuos and positive on its support then

$$
\frac{M A D(\mathbf{x})}{\sigma(\mathbf{x})}=\Phi^{-1}\left(\frac{3}{4}\right)
$$

The next result is very well known in linear algebra and it will be used to prove the equivalence of the projection depth function and the Mahalanobis distance; see, for example [27] p. 65.

Proposition 3: For $\mathbf{A}$ a positive definite matrix, and $\mathbf{b}$ a given vector, and $\mathbf{u}$ a non zero arbitrary vector,

$$
\sup _{\mathbf{u} \neq \mathbf{0}} \frac{\left(\mathbf{u}^{T} \mathbf{b}\right)^{2}}{\mathbf{u}^{T} \mathbf{A u}}=\mathbf{b}^{T} \mathbf{A}^{-1} \mathbf{b}
$$

Proposition 4: ([26] p.43) Assume that $\mathbf{X} \sim E_{d}(\boldsymbol{\mu}, \boldsymbol{\Sigma}, \psi)$ with $\operatorname{rank}(\boldsymbol{\Sigma})=r, \mathbf{B}$ is a $d \times k$ matrix and $\mathbf{v}$ is a $k \times 1$ vector, then

$$
\mathbf{v}+\mathbf{B}^{T} \mathbf{X} \sim E_{m}\left(\mathbf{v}+\mathbf{B}^{T} \mu, \mathbf{B}^{T} \mathbf{\Sigma} \mathbf{B}, \psi\right)
$$

We now state our first proposition for the case of standardized random projections.

Proposition 5: Let $\mathbf{X}_{(d \times n)}$ be a i.i.d. random sample of size $n$, where $\mathbf{x}_{i} \sim E_{d}(\boldsymbol{\mu}, \boldsymbol{\Sigma}, g)$, then:

$$
\sup _{\mathbf{u} \in \mathbb{S}^{d-1}} \frac{\left(\mathbf{u}^{T} \mathbf{x}-\boldsymbol{\mu}\left(\mathbf{u}^{T} \mathbf{X}\right)\right)^{2}}{\sigma^{2}\left(\mathbf{u}^{T} \mathbf{X}\right)}=(\mathbf{x}-\boldsymbol{\mu})^{T} \boldsymbol{\Sigma}^{-1}(\mathbf{x}-\boldsymbol{\mu})
$$

where $\mu$ is the mean and $\sigma^{2}$ is the variance.

Proof: From Prop. 4, we have $\mu\left(\mathbf{u}^{T} \mathbf{X}\right)=\mathbf{u}^{T} \boldsymbol{\mu}$ and $\sigma^{2}\left(\mathbf{u}^{T} \mathbf{X}\right)=\mathbf{u}^{T} \mathbf{\Sigma} \mathbf{u}$. Therefore

$$
\begin{aligned}
\sup _{\mathbf{u} \in \mathbb{S}^{d-1}} \frac{\left(\mathbf{u}^{T} \mathbf{x}-\mu\left(\mathbf{u}^{T} \mathbf{X}\right)\right)^{2}}{\sigma^{2}\left(\mathbf{u}^{T} \mathbf{X}\right)} & =\sup _{\mathbf{u} \in \mathbb{S}^{d-1}} \frac{\left(\mathbf{u}^{T} \mathbf{x}-\mathbf{u}^{T} \boldsymbol{\mu}\right)^{2}}{\mathbf{u}^{T} \mathbf{\Sigma} \mathbf{u}} \\
\sup _{\mathbf{u} \in \mathbb{S}^{d-1}} \frac{\left(\mathbf{u}^{T}(\mathbf{x}-\boldsymbol{\mu})\right)^{2}}{\mathbf{u}^{T} \mathbf{\Sigma} \mathbf{u}} & =\sup _{\mathbf{u} \neq \mathbf{0}} \frac{\left(\left(\frac{\mathbf{u}}{\|\mathbf{u}\|}\right)^{T}(\mathbf{x}-\boldsymbol{\mu})\right)^{2}}{\left(\frac{\mathbf{u}}{\|\mathbf{u}\|}\right)^{T} \boldsymbol{\Sigma}\left(\frac{\mathbf{u}}{\|\mathbf{u}\|}\right)}
\end{aligned}
$$

by Prop. 3 the proof is complete.

Finally, we provide the corresponding particularized result to the case of projection depth function in elliptically symmetric random variables.

Proposition 6: Let $\mathbf{X}_{(d \times n)}$ be a i.i.d. random sample of size $n$, where $\mathbf{x}_{i} \sim E_{d}(\boldsymbol{\mu}, \boldsymbol{\Sigma}, g)$ is symmetric, then:

$$
c_{g} P D(\mathbf{x} ; \mathbf{X})^{2}=(\mathbf{x}-\boldsymbol{\mu})^{T} \boldsymbol{\Sigma}^{-1}(\mathbf{x}-\boldsymbol{\mu})
$$

with $c_{g}=\left(\Phi^{-1}(3 / 4)\right)^{2}$. 
Proof:

$$
\begin{aligned}
P D(\mathbf{x} ; \mathbf{X})^{2} & =\sup _{\mathbf{u} \in S^{d-1}}\left\{\frac{\left|\mathbf{u}^{T} \mathbf{x}-\operatorname{MED}\left(\mathbf{u}^{T} \mathbf{X}\right)\right|^{2}}{\operatorname{MAD}\left(\mathbf{u}^{T} \mathbf{X}\right)^{2}}\right\} \\
& =\sup _{\mathbf{u} \in S^{d-1}}\left\{\frac{\left(\mathbf{u}^{T}(\mathbf{x}-\mu)\right)^{2}}{\mathbf{u}^{T} \Sigma \mathbf{u}}\left(\frac{\sigma\left(\mathbf{u}^{T} \mathbf{X}\right)}{\operatorname{MAD}\left(\mathbf{u}^{T} \mathbf{X}\right)}\right)^{2}\right\}
\end{aligned}
$$

and by using Propositions 2 and 5 .

$$
P D(\mathbf{x} ; \mathbf{X})^{2}\left(\Phi^{-1}\left(\frac{3}{4}\right)\right)^{2}=(\mathbf{x}-\boldsymbol{\mu})^{T} \boldsymbol{\Sigma}^{-1}(\mathbf{x}-\boldsymbol{\mu}) .
$$

Summarizing our theoretical results, projection depth function provides an order from the "center" of multidimensional data to "outlier" values. In the case of elliptically symmetric random variables, it approximates the Mahalanobis distance from the "vector mean" without any covariance matrix estimation. Indeed, it is well-known in multivariate statistics that the computation of the covariance matrix and its inverse is an ill-posed problem with the dimensionally of the data increases [28]. This is particularly pertinent in the case of hyperspectral images. In addition, the covariance matrix is very sensitive to the presence of noise in the data. Additionally, the formulation using random projections allows to include robust estimators by interchanging the couples $(\mu, \sigma)$ and (MED, MAD). This robustness is highly recommended, because the hypothesis of symmetry in the cloud representation, is too strong for natural images. We end this section by providing an implementation of the proposed approach (see Algorithm 1).

Algorithm 1 Calculate $P D(\cdot ; \mathbf{I})$ based on $k$ projections

Require: $k \geq 1$ and the data matrix $\mathbf{X}$ of $(n)$ rows and $d$ columns.

Ensure: $\mathbf{y}=P D(\cdot ; \mathbf{X})$

1: $\mathbf{y}=\mathbf{0}_{n_{1} \times 1}$

2: for all $i=1$ to $k$ do

3: $\quad \mathbf{r}=\operatorname{RANDN}(d, 1)$ (Random Gaussian generation).

4: $\quad \mathbf{r}=\mathbf{r} /\|\mathbf{r}\|\left(\right.$ Random number in $\left.\mathbb{S}^{d-1}\right)$.

5: $\mathbf{p}=\mathbf{X r}$ (Random projection).

6: medi $=$ MEDIAN $(\mathbf{p})$ (Median of the Random projection).

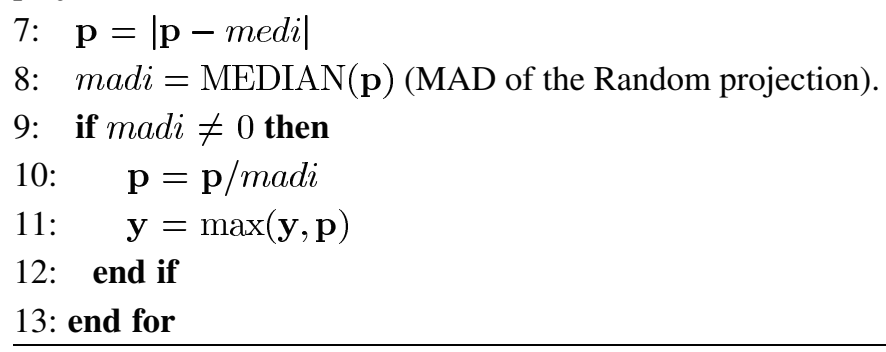

\section{Multivariate Vector Morphology Using PROJECTION DEPTH FUNCTIONS}

The rationale behind our formulation of MM for multivariate images is to use projection depth functions in the vector space to produce a vector ordering. As discussed above, statistical depth functions provide from the "deepest" point a "center-outward" ordering of multidimensional data. According to the taxonomy of Barnet's orderings [6], it seems natural to say that statistical depth function involves a P-ordering. However, according to the result provided in previous section, statistical depth function can be interpreted as a robust estimate, up to a multiplicative constant, of a distance from the center of the image vector values and consequently it can be considered also as a R-ordering. In fact, from our viewpoint, any P-ordering based on extremeness is essentially a R-ordering according to a particular centrality measure.

Given a vector image $\mathbf{I} \in \mathcal{F}(\mathrm{E}, \mathbb{F})$, let $\mathbf{X}_{\mathbf{I}}$ be the set of vector values of the image, which can be viewed as a cloud of points in $\mathbb{F}$. Fig. 1 shows an example of color image $\mathbf{I}$, its representation as points $\mathbf{X}_{\mathbf{I}}$, and the image of the associated depth function $P D(\cdot ; \mathbf{I})$. The ordering for two pixel vectors is given by $\mathbf{x}_{1}<\mathbf{x}_{2} \leftrightarrow P D\left(\mathbf{x}_{1} ; \mathbf{I}\right)<P D\left(\mathbf{x}_{2} ; \mathbf{I}\right)$. That is an ordering based on a data-adapted function and in such a way that the interpretation of supremum and infimum operations is known a priori, because in the discrete setting, max values can be associated with "outlier" pixels in the high-dimensional space and min are "central" pixels in $\mathbb{R}^{d}$ space. Projection depth function can be computed for any image, but is the order associated to the statistical depth function appropriate for any image? Or in other terms, in which cases the notions of "outlier" pixels and "central" pixels make sense? We consider that for such images the assumption of existence of a background/foreground representation is required. Formally we could express the assumption of background/foreground representation in this way. Given a vector image $\mathbf{I}: E \rightarrow \mathbb{F}$, the sub-space of vector values $\mathbf{X}_{\mathbf{I}}$ has a decomposition $\mathbf{X}_{\mathbf{I}}=\left\{\mathbf{X}_{B(\mathbf{I})}, \mathbf{X}_{F(\mathbf{I})}\right\}$ such that $\mathbf{X}_{B(\mathbf{I})} \cap \mathbf{X}_{F(\mathbf{I})}=\emptyset$ and $\operatorname{card}\left\{\mathbf{X}_{B(\mathbf{I})}\right\}>\operatorname{card}\left\{\mathbf{X}_{F(\mathbf{I})}\right\}$. Roughly speaking, the assumption means: (1) the image has two main components: the background and the foreground; (2) There are more pixels in the background than in the foreground. We notice that there is no hypothesis about the multivariate or spatial distribution of the background $\mathbf{X}_{B(\mathbf{I})}$ and the foreground $\mathbf{X}_{F(\mathbf{I})}$.

The theoretical framework of the proposed morphological operators roots in the notions of $h$-ordering and $h$-adjunctions introduced in [29]. So let us start by a reminder of the main results from [29] useful for our approach.

\section{A. h-Ordering and h-Adjunctions}

Theoretical formulation of mathematical morphology is nowadays phrased in terms of complete lattices and operators defined on them. For a detailed exposition on complete lattice theory in MM, we refer to [2]. A space $\mathcal{L}$ endowed with a partial order $\leq$ is called a complete lattice, denoted $(\mathcal{L}, \leq)$ if every subset $\mathcal{M} \subseteq \mathcal{L}$ has both supremum (join) $\bigvee \mathcal{M}$ and infimum (meet) $\bigwedge \mathcal{M}$. Let $\mathrm{R}$ be a nonempty set and assume that $\mathcal{L}$ a complete lattice. Furthermore, let $h: \mathrm{R} \rightarrow \mathcal{L}$ be a surjective mapping. Define an equivalence relation $=_{h}$ on R as follows: $\mathrm{r}={ }_{h} \mathrm{r}^{\prime} \Leftrightarrow h(\mathrm{r})=h\left(\mathrm{r}^{\prime}\right) \forall \mathrm{r}, \mathrm{r}^{\prime}$. As it was defined in [29], we refer by $\leq_{h}$ the $h$-ordering given by the following relation on $\mathrm{R}$

$$
\mathrm{r} \leq_{h} \mathrm{r}^{\prime} \Leftrightarrow h(I) \leq h\left(\mathrm{r}^{\prime}\right), \quad \forall \mathrm{r}, \mathrm{r}^{\prime} \in R
$$

Note that $\leq_{h}$ preserves reflexivity ( $\left.\mathrm{r} \leq_{h} \mathrm{r}\right)$ and transitivity $\left(r_{1} \leq_{h} \mathrm{r}_{2}\right.$ and $\left.\mathrm{r}_{2} \leq_{h} \mathrm{r}_{3} \Rightarrow \mathrm{r}_{1} \leq_{h} \mathrm{r}_{3}\right)$. However, $\leq_{h}$ is not 
a partial ordering because $\mathrm{r} \leq_{h} \mathrm{r}^{\prime}$ and $\mathrm{r}^{\prime} \leq_{h} \mathrm{r}$ implies only that $\mathrm{r}={ }_{h} \mathrm{r}^{\prime}$ but not $\mathrm{r}=\mathrm{r}^{\prime}$.

An operator $\psi: \mathrm{R} \rightarrow \mathrm{R}$ is $h$-increasing if $\mathrm{r} \leq_{h} \mathrm{r}^{\prime} \mathrm{im}$ plies that $\psi(\mathrm{r}) \leq_{h} \psi\left(\mathrm{r}^{\prime}\right)$. Additionally, since $h$ is surjective, an equivalence class is defined by $\mathcal{L}[r]=\{t \in \mathrm{R} \mid h(t)=r\}$. The Axiom of Choice [29] implies that there exist mappings $h^{\leftarrow}: \mathcal{L} \rightarrow \mathrm{R}$ such that $h h^{\leftarrow}(r)=r$, for $r \in \mathcal{L}$. Unless $h$ is injective, there exist more than one such $h^{\leftarrow}$ mappings: $h^{\leftarrow}$ is called the semi-inverse of $h$. Note that $h^{\leftarrow} h$ is not the identity mapping in general (but $h^{\leftarrow} h={ }_{h}$ id). However, we have that for any $h$-increasing $\psi: \mathrm{R} \rightarrow \mathrm{R}$ the result $\psi h^{\leftarrow} h=_{h} \psi$ and hence $h \psi h \leftarrow h=h \psi$. Let us introduce $\widetilde{\psi}$ the operator associated to $\psi$ in the lattice $\mathcal{L}$. A mapping $\psi: \mathrm{R} \rightarrow \mathrm{R}$ is $h$-increasing if and only there exists an increasing mapping $\widetilde{\psi}: \mathcal{L} \rightarrow \mathcal{L}$ such that $\widetilde{\psi} h=h \psi$. The mapping $\widetilde{\psi}$ is uniquely determined by $\psi$ and can be computed from

$$
\widetilde{\psi}=h \psi h^{\leftarrow}
$$

We can now define the $h$-erosion and $h$-dilation. Let $\varepsilon, \delta$ : $\mathrm{R} \rightarrow \mathrm{R}$ be two mappings with the property

$$
\delta(\mathrm{r}) \leq_{h} \mathrm{r}^{\prime} \Leftrightarrow \mathrm{r} \leq_{h} \varepsilon\left(\mathrm{r}^{\prime}\right), \quad \forall \mathrm{r}, \mathrm{r}^{\prime} \in \mathrm{R}
$$

then the pair $(\varepsilon, \delta)$ is called an $h$-adjunction. Moreover, let $(\varepsilon, \delta)$ be $h$-increasing mappings on $\mathrm{R}$, and let $\varepsilon \mapsto^{h} \widetilde{\varepsilon}, \delta \mapsto^{h} \widetilde{\delta}$. Then $(\varepsilon, \delta)$ is an $h$-adjunction on $\mathbf{R}$ if and only if $(\widetilde{\varepsilon}, \widetilde{\delta})$ is an adjunction on the lattice $\mathcal{L}$. Therefore a mapping $\delta$ (resp. $\varepsilon$ ) on $\mathrm{R}$ is called $h$-dilation (resp. $h$-erosion) if $\delta$ (resp. $\widetilde{\varepsilon}$ ) is a dilation (resp. erosion) on $\mathcal{L}$. $h$-adjunctions inherit a large number of properties from ordinary adjunctions between complete lattices. Assume that $(\varepsilon, \delta)$ is an $h$-adjunction then

$$
\gamma=\delta \varepsilon \leq_{h} \text { id } \leq_{h} \varphi=\varepsilon \delta .
$$

Hence, $\gamma$ is $h$-anti-extensive and $\phi$ is $h$-extensive. The operator $\gamma$ on $\mathrm{R}$ is called $h$-opening if the operator $\widetilde{\gamma}$ on $\mathcal{L}$ determined by $\gamma \mapsto^{h} \widetilde{\gamma}$ is an opening. The operator $\gamma$ is also $h$-increasing and satisfies $\gamma \gamma={ }_{h} \gamma(h$-idempotency). The $h$-closing is similarly defined.

\section{B. Vector Images and Depth h-Mapping}

The previous theoretical results can be particularized to the case of vector images using projection depth.

For multivariate images $\mathbf{I}: \mathrm{E} \rightarrow \mathbb{F}$, as color or hyperspectral ones, pixel values are vectors defined in $\mathbb{F}=\mathbb{R}^{d}$. Consequently the main challenge to build complete lattice structures is to define a mapping

$$
h: \rightarrow \mathbb{F} \rightarrow \mathcal{L},
$$

where $\mathcal{L}$ can be the lattice of the extended real line, i.e., $\mathcal{L}=$ $(\overline{\mathbb{R}}, \leq)$, with $\overline{\mathbb{R}}=\mathbb{R} \cup\{-\infty,+\infty\}$ and $\leq$ as the "less than or equal to" operation (the natural partial ordering). Furthermore the composition of $\mathbf{I}$ and $h$ will be denoted by $h(\mathbf{I}): \mathbf{E} \rightarrow$ $\mathcal{L}$. According to the previous subsection, once the mapping $h$ has been established, the morphological vector operators can be defined as $h$-adjunctions.
Given a multivariate vector image $\mathbf{I} \in \mathcal{F}(\mathrm{E}, \mathbb{F})$, its $h$-depth mapping is defined as

$$
h_{\mathbf{I}}(\mathbf{x})=P D\left(\mathbf{x} ; \mathbf{X}_{\mathbf{I}}\right)
$$

Therefore, the ordering $\leq_{h_{\mathrm{I}}}$ generated by the projection depth function yields morphological operators which can be interpreted as follows: low values in $\mathcal{L}$ induced by $h_{\mathbf{I}}(\mathbf{x})$ correspond to pixels close to the "background" (median vector) and high values in $\mathcal{L}$ coincide with "foreground" (outlier vectors). That is coherent with binary and gray-level morphology, where high gray-levels are associated to the objects (foreground) and low gray-levels to the background.

We have now the ingredients to formulate the corresponding multivariate vector erosion and dilation We limit here our developments to the flat operators, i.e., the structuring elements are planar shapes. The non-planar structuring functions are defined by weighting values on their support [31]. The $h$-depth erosion $\varepsilon_{\mathrm{SE}, h_{\mathbf{I}}}(\mathbf{I})$ and $h$-depth dilation $\delta_{\mathrm{SE}, h_{\mathbf{I}}}(\mathbf{I})$ of an image $\mathbf{I}$ at pixel $x \in \mathrm{E}$ by the structuring element $\mathrm{SE} \subset \mathrm{E}$ are the two mappings $\mathcal{F}(\mathrm{E}, \mathbb{F}) \rightarrow \mathcal{F}(\mathrm{E}, \mathbb{F})$ defined respectively

$$
h_{\mathbf{I}}\left(\varepsilon_{\mathrm{SE}, h_{\mathbf{I}}}(\mathbf{I})(x)\right)=\widetilde{\varepsilon}_{\mathrm{SE}}\left(h_{\mathbf{I}}(\mathbf{I})\right)(x),
$$

and

$$
h_{\mathbf{I}}\left(\delta_{\mathrm{SE}, h_{\mathbf{I}}}(\mathbf{I})(x)\right)=\widetilde{\delta}_{\mathrm{SE}}\left(h_{\mathbf{I}}(\mathbf{I})\right)(x),
$$

where $\widetilde{\varepsilon}_{\mathrm{SE}}(I)$ and $\widetilde{\delta}_{\mathrm{SE}}(I)$ are the standard numerical flat erosion and dilation of image $I \in \mathcal{F}(\mathrm{E}, \mathcal{L})$ :

$$
\begin{aligned}
& \widetilde{\varepsilon}_{\mathrm{SE}}(I)(x)=\left\{I(y): I(y)=\bigwedge[I(z)], z \in \mathrm{SE}_{x}\right\} \\
& \widetilde{\delta}_{\mathrm{SE}}(I)(x)=\left\{I(y): I(y)=\bigvee[I(z)], z \in \hat{\mathrm{SE}}_{x}\right\}
\end{aligned}
$$

with $\mathrm{SE}_{x}$ being the structuring element centered at point $x$ and $\hat{\mathrm{SE}}$ is the reflected structuring element. If the inverse mapping $h_{\mathbf{I}}^{-1}$ is defined, the $h$-depth erosion and dilation can be explicitly written as:

$$
\varepsilon_{\mathrm{SE}, h_{\mathbf{I}}}(I)(x)=h_{\mathbf{I}}^{-1}\left(\widetilde{\varepsilon}_{\mathrm{SE}}\left(h_{\mathbf{I}}(\mathbf{I})\right)\right)(x),
$$

and

$$
\delta_{\mathrm{SE}, h_{\mathbf{I}}}(I)(x)=h_{\mathbf{I}}^{-1}\left(\widetilde{\delta}_{\mathrm{SE}}\left(h_{\mathbf{I}}(\mathbf{I})\right)\right)(x) .
$$

Of course, the inverse $h^{-1}$ only exists if $h$ is injective. Theoretically, this is not guaranteed for $h_{\mathbf{I}}$ since two different vectors $\mathbf{x}_{1}$ and $\mathbf{x}_{2}$ can have the same projection depth; i.e., $\mathbf{x}_{1} \neq \mathbf{x}_{2}$ but $P D\left(\mathbf{x}_{1} ; \mathbf{X}_{\mathbf{I}}\right)=P D\left(\mathbf{x}_{2} ; \mathbf{X}_{\mathbf{I}}\right)$. We can impose in practice the invertibility of $h_{\mathbf{I}}$ by considering a lexicographic ordering for equivalence class $\mathcal{L}[\mathbf{x}]$. In fact, this solution involves a structure of total ordering which allows to compute directly the $h$-depth erosion and dilation without using the inverse mapping, i.e.,

$$
\varepsilon_{\mathrm{SE}, h_{\mathbf{I}}}(\mathbf{I})(x)=\left\{\mathbf{I}(y): \mathbf{I}(y)=\bigwedge_{h_{\mathbf{I}}}[\mathbf{I}(z)], z \in \mathrm{SE}_{x}\right\},
$$




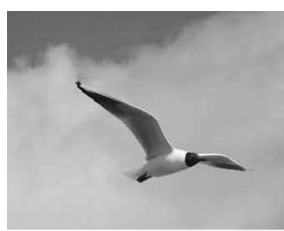

(a)

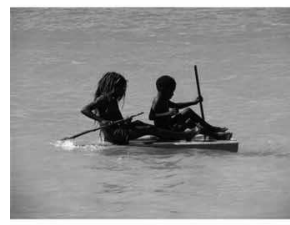

(g)

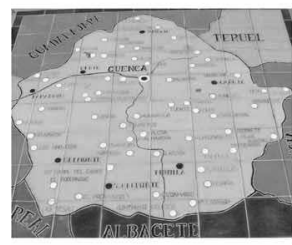

(m)

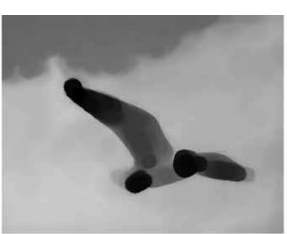

(b)

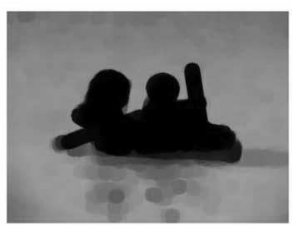

(h)

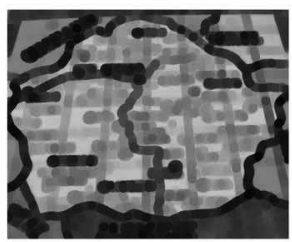

(n)

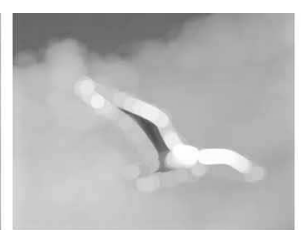

(c)

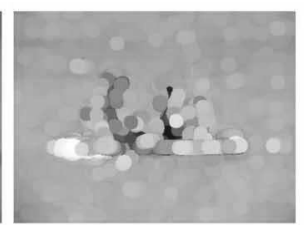

(i)

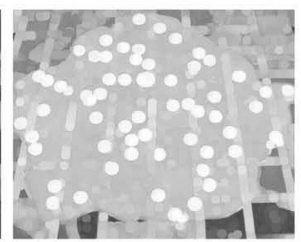

(o)

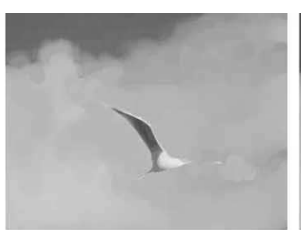

(d)

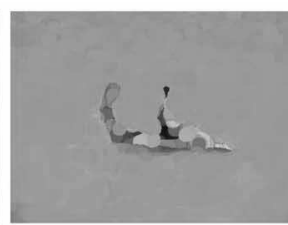

(j)

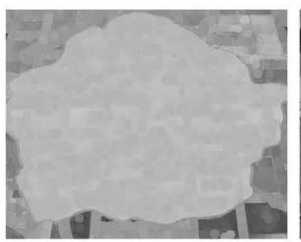

(p)

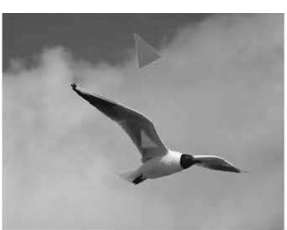

(e)

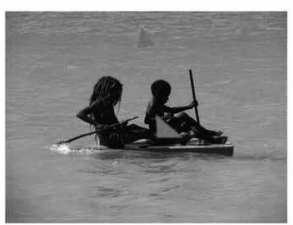

(k)

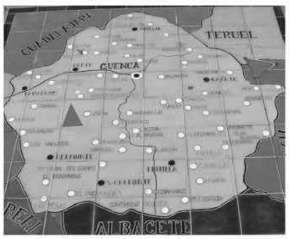

(q)

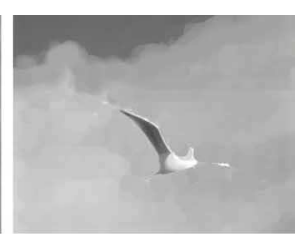

(f)

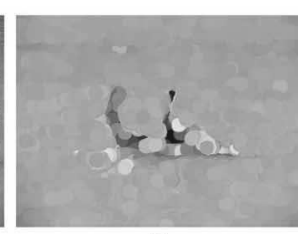

(1)

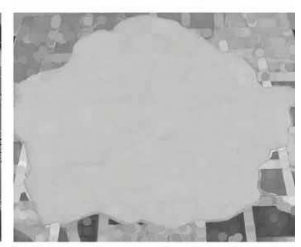

(r)

Fig. 2. Erosions by a disk of size 10 in the family of orders proposed by Barnet [6] and recent approaches from [9] and [30]. C-ordering uses the priority red $>$ green $>$ blue. Proposed P-ordering is illustrated in (e)-(k)-(q) with $k=1000$ random projections. Supervised ordering from [30] is calculated by SVM with background/foreground sets given by green/red triangles in $(\mathrm{e})(\mathrm{k})(\mathrm{q})$ respectively. Erosion in the ordering induced by the proposed P-ordering follows the physical meaning of the transformation, i.e., diminution in the size of the objects is produced. The ordering does not require a training set as supervised ordering (f)-(l)-(r). However, this intrinsic ordering is based on dichotomy background and foreground (See text for more details). (a) Original; (b) M-ordering; (c) C-ordering [9]; (d) P-ordering; (e) Training set; (f) supervised ordering [30]; (g) original; (h) M-ordering; (i) C-ordering [9]; (j) P-ordering; (k) Training set; (l) supervised ordering [30]; (m) original; (n) M-ordering; (o) C-ordering [9]; (p) P-ordering; (q) Training set; (r) supervised ordering [30].

and

$$
\delta_{\mathrm{SE}, h_{\mathbf{I}}} \mathbf{I}(x)=\left\{\mathbf{I}(y): \mathbf{I}(y)=\bigvee_{h_{\mathbf{I}}}[\mathbf{I}(z)], z \in \operatorname{SE} t_{x}\right\},
$$

where $\bigwedge_{h_{\mathrm{I}}}$ and $\bigvee_{h_{\mathrm{I}}}$ are respectively the infimum and supremum according to the ordering $\leq_{h_{\mathrm{I}}}$, induced for the projection depth function $P D\left(\mathbf{x} ; \mathbf{X}_{\mathbf{I}}\right)$ and completed with a lexicographic ordering in $\mathbb{F}$. Starting from the $h$-depth adjunction $\left(\varepsilon_{\mathrm{SE}, h_{\mathbf{I}}}(\mathbf{I}), \varepsilon_{\mathrm{SE}, h_{\mathbf{I}}}(\mathbf{I})\right)$, all the morphological filters such as the opening and closing have their $h$-depth counterpart, e.g., the $h$-depth opening and closing are defined as

$$
\gamma_{\mathrm{SE}, h_{\mathbf{I}}}(\mathbf{I})=\delta_{\mathrm{SE}, h_{\mathbf{I}}}\left(\varepsilon_{\mathrm{SE}, h_{\mathbf{I}}}(\mathbf{I})\right), \phi_{\mathrm{SE}, h_{\mathbf{I}}}(\mathbf{I})=\varepsilon_{\mathrm{SE}, h \mathbf{I}}\left(\delta_{\mathrm{SE}, h \mathbf{I}}(\mathbf{I})\right)
$$

Similarly, geodesic operators as opening by reconstruction [7], $\gamma_{\mathrm{SE}}^{\infty}(\mathbf{I})$, can be also naturally extended to multivariate images.

\section{Properties}

$h$-depth vector erosion and dilation inherit the standard algebraic properties of morphological operators [2], [31] since they fit into the theory of $h$-adjunctions. Nevertheless, some remarks about their particular behavior are useful for practical applications.

Filtering effects. Multivariate morphological operators defined using $h$-depth adjunction have the classical filtering properties [7]. Namely, the erosion shrinks the structures which pixel values distant to the center in the vector dimensional space; "spatial peaks" thinner than the structuring element disappear by taking the value of neighboring pixels with a vector value close to the "background." As well, it expands the structures which have a vector value close to "foreground." Fig. 2 illustrates these effects in comparison with marginal, conditional, reduced order by saturation [32] and supervised ordering [30]. Dilation produces the dual effects, enlarging the regions having values close to the outliers and contracting the background. The other morphological operators are naturally interpreted as products of dilations and erosions. Concerning the product operators, opening (closing) is an idempotent and anti-extensive (extensive) operator, which removes foreground (background) objects that are smaller than the structuring element, leaving intact the structures invariant to the structuring element.

From the image analysis viewpoint, we can consider that the $h$-depth erosion/dilation, and all the associated operators, are unsupervised transformations, in the sense that ordering is intrinsically adapted to the image without giving any training set of vectors for the background and foreground. We refer to our recent study [30] for the formulation of a supervised framework.

Duality. The notion of duality by complement in gray level images $I \in \mathcal{F}(\mathrm{E}, \mathcal{L})$ allows to compute the dilation using the erosion operator, i.e., $\widetilde{\delta}_{\mathrm{SE}}(I)=\complement \widetilde{\varepsilon}_{\mathrm{SE}}(\complement I)$, where $\complement I=-I$. The ordering function $h_{\mathbf{I}}(\mathbf{x}) \leq h_{\mathbf{I}}(\mathbf{f})$ for all $\mathbf{x} \in \mathbb{F}$ and some $\mathbf{f} \in \mathbf{X}_{F(\mathbf{I})}$, and equivalently, $h_{\mathbf{I}}(\mathbf{x}) \geq h_{\mathbf{I}}(\mathbf{b})$ for all $\mathbf{x} \in \mathbb{F}$ and some $\mathbf{b} \in \mathbf{X}_{B(\mathbf{I})}$. Hence, the smallest element of the vector space belongs to the "background" and the largest to the "foreground," i.e., $\perp_{\mathbb{F}} \in \mathbf{X}_{B(\mathbf{I})}$ and $T_{\mathbb{F}} \in \mathbf{X}_{F(\mathbf{I})}$. We have therefore a qualitative dual role played by the background and foreground of the image. However, the quantitative duality does not involved an involution on $\mathbb{F}$ : projection depth is invariant to the complement of the vector coordinates. The duality by 


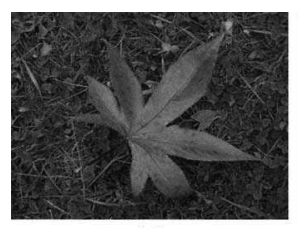

(a)

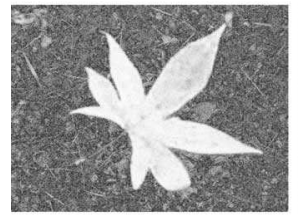

(d)

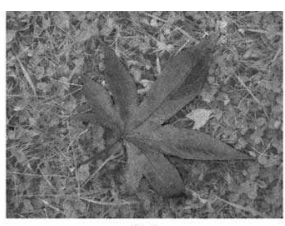

(b)

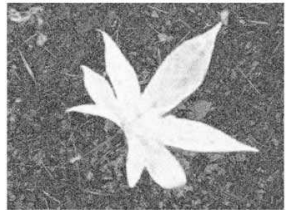

(e)

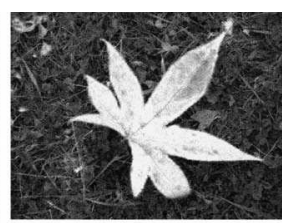

(c)

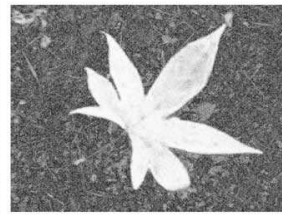

(f)
Fig. 3. Projection depth function is invariant to affine transformation in $\mathbb{R}^{d}$. (a)-(c) are obtained by affine transformations in $\mathbb{R}^{3}$ of Fig. 1(a). (d)-(f) show approximated $P D(\cdot ; k, \mathbf{I})$, with $k=1000$ random projections.

complement appears in the $h$-depth mapping which involves, $h_{\mathbf{I}}\left(\delta_{\mathrm{SE}, h_{\mathbf{I}}}(\mathbf{I})(x)\right)=-\widetilde{\varepsilon}_{\mathrm{SE}}\left(-h_{\mathbf{I}}(\mathbf{I})\right)(x)$ and consequently

$$
\delta_{\mathrm{SE}, h_{\mathbf{I}}}(\mathbf{I})(x)=\varepsilon_{\mathrm{SE},-h_{\mathbf{I}}}(\mathbf{I})(x) .
$$

Invariance. From its original formulation MM is contrast invariant due to its basic operators are based on rank filters [7]. The representation of the image as a topographic map is a key point of the contrast invariance properties of MM in gray scale images [33]. Contrast invariant is an interesting property linked to the fact that image gray level is not an absolute data, since in many cases the pixel values depend of the acquisition circumstances. For instance, the contrast depends on the type of the camera and illuminations conditions of the scene. Mathematically, we say that the $h$-ordering is contrast invariant if for every continuos contrast change $\mathbf{b} \in \mathbb{R}^{d}, \mathbf{x}_{1} \leq_{h_{\mathrm{I}}} \mathbf{x}_{2} \Rightarrow$ $\mathbf{x}_{1} \leq_{h_{\mathbf{I}+\mathbf{b}}} \mathbf{x}_{2}$, for all $\mathbf{x}_{1}, \mathbf{x}_{2}, \mathbf{b} \in \mathbb{R}^{d}$ and $\forall \mathbf{I} \in \mathcal{F}\left(\mathrm{E}, \mathbb{R}^{d}\right)$, where $h_{\mathbf{I}+\mathbf{b}}$ denotes the $h$-mapping calculated from $\mathbf{I}+\mathbf{b}$. From definition (2), it is easy to see $P D\left(\mathbf{x} ; \mathbf{X}_{\mathbf{I}}\right)=P D\left(\mathbf{x} ; \mathbf{X}_{\mathbf{I}}+\right.$ $\mathbf{b}), \forall \mathbf{x}, \mathbf{b} \in \mathbb{R}^{d}$, and its approximated version (3) is contrast invariance when the number of random projections $k$ is large enough, i.e., $P D\left(\cdot ; k, \mathbf{X}_{\mathbf{I}}\right)=P D\left(\cdot ; k, \mathbf{X}_{\mathbf{I}}+\mathbf{b}\right), \forall \mathbf{x} \in \mathbb{R}^{d}$, when $k$ tend to $\infty$. Thus, the contrast invariance property is not automatic guaranteed, but it depends on the number of projections in the expression (3).

Another more general suitable property is the invariance to affine transformation. An $h$-ordering is said to be invariant to affine transformation if, for every $\mathbf{A} \in \mathbb{R}^{d \times d}$ definite positive matrix and $\mathbf{b} \in \mathbb{R}^{d}$ a vector, $h$-ordering is invariant to the transformation defined by $\Gamma(\mathbf{I})=\mathbf{A X}_{\mathbf{I}}+\mathbf{b}$, i.e., $\mathbf{x}_{1} \leq_{h_{\mathbf{I}}} \mathbf{x}_{2} \Rightarrow$ $\mathbf{x}_{1} \leq_{h_{\Gamma(\mathbf{I})}} \mathbf{x}_{2}$, for all $\mathbf{x}_{1}, \mathbf{x}_{2} \in \mathbb{R}^{d}$. Affine transformations includes rotation and scaling, but also shearing. From [15], (2) is affine invariant for $\mathbf{X}$ in the family of symmetric distributions, i.e., $P D(\cdot ; \mathbf{X})=P D(\cdot ; \mathbf{A X})$ for $\mathbf{A} \in \mathbb{R}^{d \times d}$ a definite positive matrix. Nevertheless, there is not guarantee of a image $\mathbf{I}$ with background/foreground representation has symmetric distribution. However, experimental results shows that proposed ordering is robust to affine transformation in the vector space $\mathbb{R}^{d}$. This situation is illustrated in Fig. 3 .

Local knowledge. Given an image I parameterized for its spatial support, $\mathbf{I}_{\mathrm{E}}: \mathrm{E} \rightarrow \mathbb{F}$, and a subset in its spatial sup-
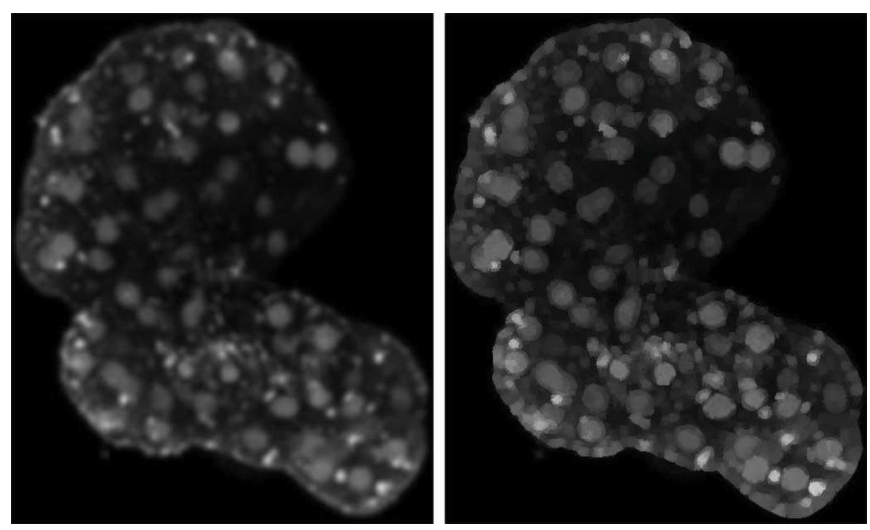

Fig. 4. Edge enhancement of $\mathbf{I}$ using toggle mapping $\tau_{h}(\mathbf{I})$ in the proposed ordering. Source: http://www.cellimagelibrary.org/

port, $E^{\prime} \subset \mathrm{E}$, the depth functions associated of both images are not equivalent, i.e, $h_{\mathbf{I}_{\mathrm{E}}} \neq h_{\mathbf{I}_{\mathrm{E}^{\prime}}}$. However, the local knowledge property [31] is preserved if and only if the depth function is calculated using the whole available image in $\mathrm{E}$.

\section{APPLICATIONS}

This section presents three application examples that utilize MM operators in the induced ordering by random projection depth. The aim is to demonstrate the impact of this unsupervised ordering in standard morphological operators for object filtering and segmentation in vector images. The definition $h$-depth ordering can be applied to multivariate images, allowing to use "any" morphological operator for color, multispectral and hyperspectral images. Theoretically, convergence of iterative algorithms is guarantied if the $h$-depth ordering induced a total order, once completed with lexicographic order. In these experiments, the number of random projections in (3) have been fixed to $k=1000$. An optimal parameter selection can be done over $k$, however that is beyond the scope of this paper. The first application concerns edge enhancement in multivariate images and is based on shock filters [7], [31].

\section{A. Image Enhancement}

Given an image I, an two transformation $\Psi(\mathbf{I}) \leq_{h_{\mathbf{I}}} \mathbf{I} \leq_{h_{\mathbf{I}}}$ $\Upsilon(\mathbf{I})$ the shock filter is defined as follows

$$
\tau_{h_{\mathbf{I}}}(\mathbf{I})= \begin{cases}\Psi_{h_{\mathbf{I}}}(\mathbf{I}) & \text { if } \Delta_{h_{\mathbf{I}}}(\mathbf{I}, \Psi, \Upsilon)<0 \\ \Upsilon_{h_{\mathbf{I}}}(\mathbf{I}) & \text { if } \Delta_{h_{\mathbf{I}}}(\mathbf{I}, \Psi, \Upsilon)>0 \\ \mathbf{I} & \text { otherwise }\end{cases}
$$

where $\Delta_{h_{\mathbf{I}}}(\mathbf{I}, \Psi, \Upsilon)=h_{\mathbf{I}}(\mathbf{I}-\Psi(\mathbf{I}))-h_{\mathbf{I}}(\Upsilon(\mathbf{I})-\mathbf{I})$ is the morphological Laplacian of the original image, based on $\Psi$ and $\Upsilon$ in the domain of $h$-depth function. For gray scale images, in the particular case of $h$ as the identity function, $\Psi_{h_{\mathbf{I}}}(\mathbf{I})=\varepsilon_{\mathrm{SE}, h_{\mathbf{I}}}(\mathbf{I})$, $\Upsilon_{h_{\mathbf{I}}}(\mathbf{I})=\delta_{\mathrm{SE}, h_{\mathbf{I}}}(\mathbf{I})$, and SE as the unitary ball, we have the classical shock filter introduced by Kramer [34]. It is based on the idea of using a dilation process near a local maximum and an erosion process around a local minimum. The toggle mapping $\tau_{h_{\mathrm{I}}}$ enhances images edges detected by differences in the projection depth function, i.e., background/foreground transitions. The enhanced image tends to be piecewise constant due to morphological operators and preserves the original information in pixels where the edge detector is ambiguous (otherwise case 


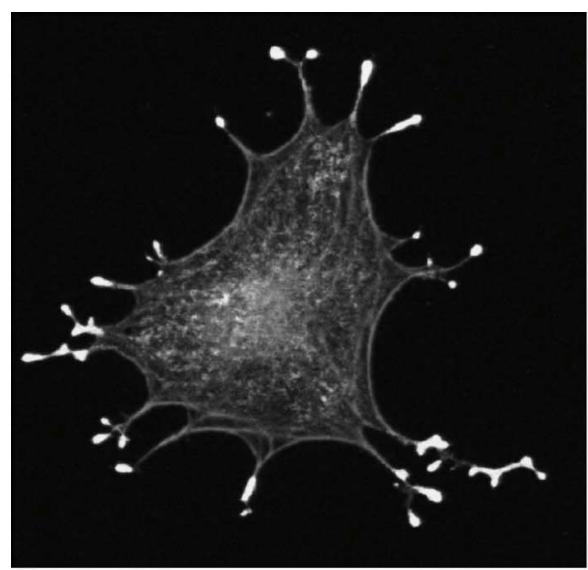

(a)

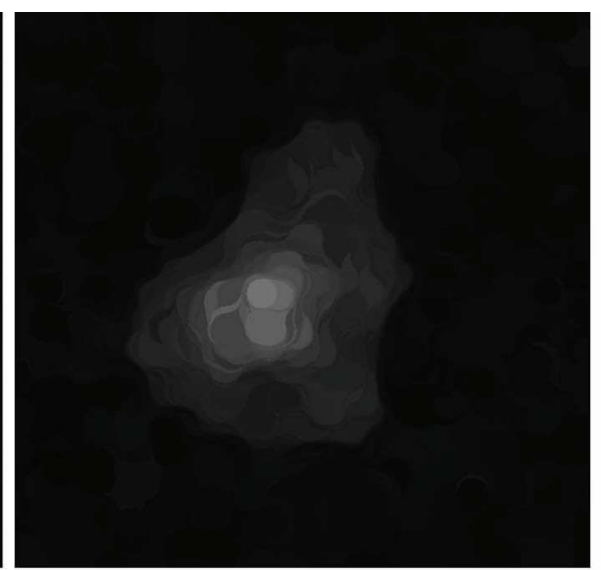

(b)

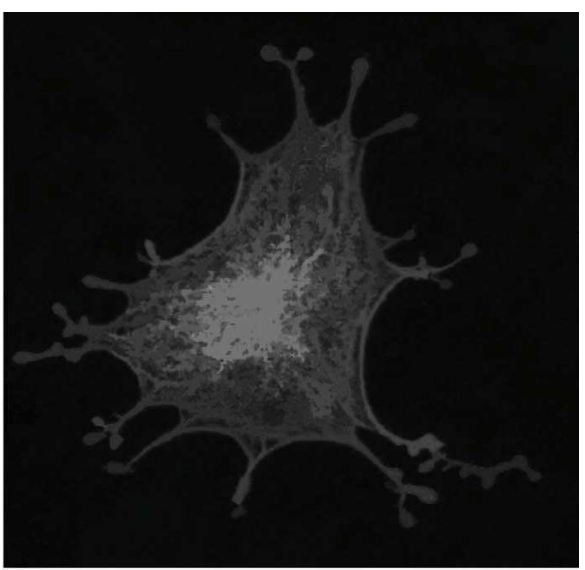

(c)

Fig. 5. Original (I), marker (M) and simplification by $h$-depth vector leveling $\Lambda_{h \mathbf{I}}(\mathbf{I}, \mathbf{M})$. The marker $\mathbf{M}$ is the product of a $h$-depth closing followed by an $h$-depth opening with the $\mathrm{SE}$ is a disk of size 10. (a) $\mathbf{I}$; (b) $\mathbf{M}=\gamma_{\mathrm{SE}, h_{\mathbf{I}}}\left(\phi_{\mathrm{SE}, h_{\mathbf{I}}}(\mathbf{I})\right) ;$ (c) $\Lambda_{h_{\mathbf{I}}}(\mathbf{I}, \mathbf{M})$.

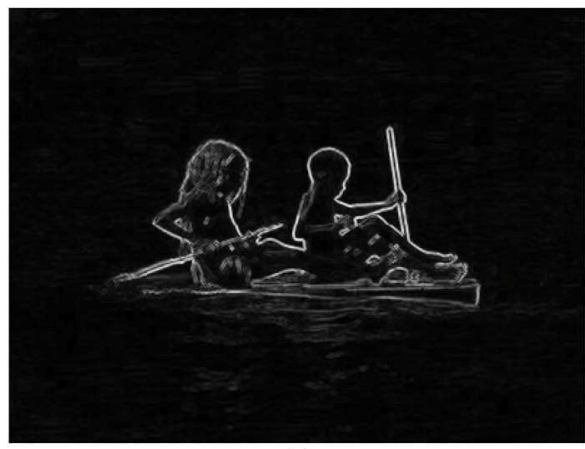

(a)

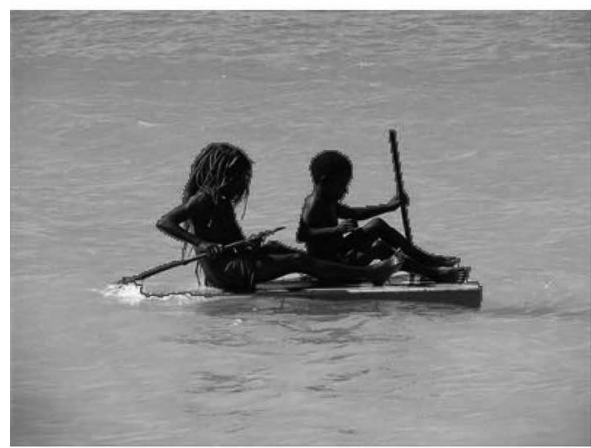

(d)

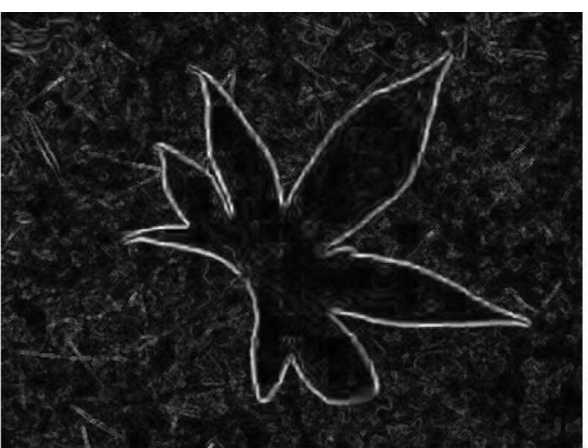

(b)

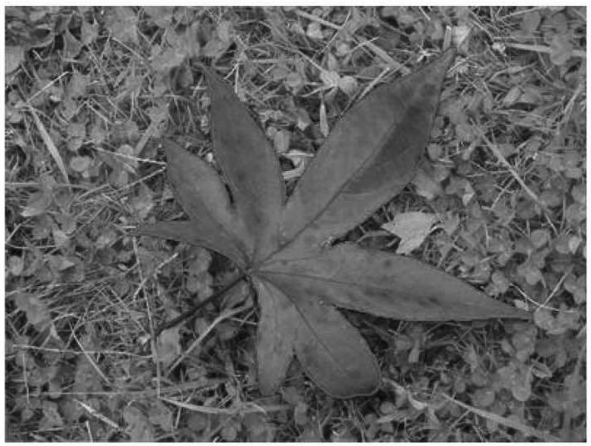

(e)

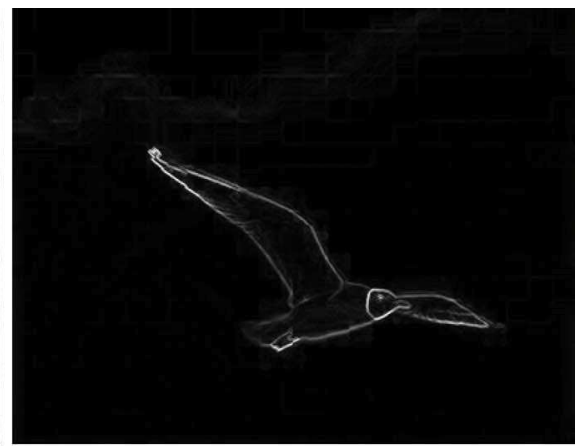

(c)

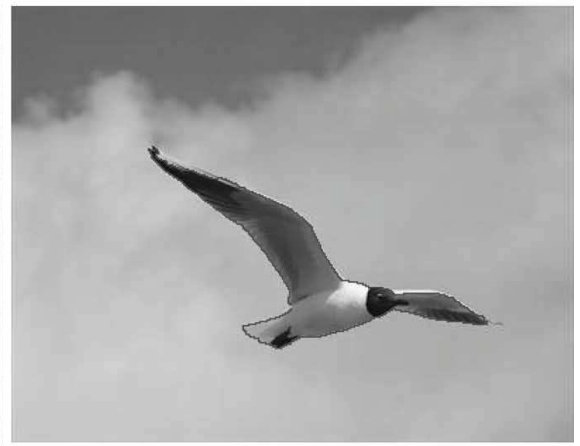

(f)

Fig. 6. $h$-depth gradient and segmentation by using watershed transformation (in red), where markers are calculated by selecting the minima of strong dynamics in $h$-depth gradient, with $t=.5$. (a) $\Delta_{h}(\mathbf{I})$; (b) $\Delta_{h}$ (I); (c) $\Delta_{h}(\mathbf{I})$; (d) WS $(\mathbf{I}, t)$; (e) WS(I,t); (f) WS $(\mathbf{I}, t)$.

in (21)). Additionally, the vector formulation allows to perform edge enhancement without include false colors during the procedure. Fig. 4 presents an illustrative example to show how the toggle mapping works for a vector image.

\section{B. Image Simplification}

The task of image simplification is the subject of various approaches and applications [35], [36]. The aim is to produce from an initial image, an approximated version with is simpler in some sense. In the list of MM operators, the morphological leveling attempts to produce flat zones for an original image $\mathbf{I}$ from a given marker image $\mathbf{M}$, consequently, simpler according to the norm of the gradient, but preserving the main object according to the marker M. The idea of such a filter goes back to Matheron [37] and Meyer [38]. In the induced order produced by a $h$-depth function, an image $\mathbf{J}$ is a leveling of the image $\mathbf{I}$, denoted by $\mathbf{J} \in \Lambda(\mathbf{I})$, iff $\forall(p, q) \in \mathrm{E} \times \mathrm{E}$ neighbors:

$$
\mathbf{J}(p)>_{h_{\mathbf{I}}} \mathbf{J}(q) \Rightarrow \mathbf{I}(p) \leq_{h_{\mathbf{I}}} \mathbf{J}(p) \text { and } \mathbf{J}(q) \geq_{h_{\mathbf{I}}} \mathbf{I}(q)
$$

The criterion also gives the clue to the algorithm for constructing a leveling. The function $\mathbf{J}$ is modified until the criterion is satisfied, on $K^{+}=\left\{x\right.$ s.t. $\left.\mathbf{J}(x)<_{h_{\mathbf{I}}} \mathbf{I}(x)\right\}$, $\mathbf{J}$ is replaced by $\mathbf{I} \wedge \wedge_{h_{\mathbf{I}}} \delta_{\mathrm{SE}, h_{\mathbf{I}}}(\mathbf{J})$ and on $K^{-}=\left\{x\right.$ s.t. $\left.\mathbf{J}(x)>_{h_{\mathbf{I}}} \mathbf{I}(x)\right\}$, J, by $\mathbf{I} \vee_{h_{\mathbf{I}}} \varepsilon_{\mathrm{SE}, h_{\mathbf{I}}}(\mathbf{J})$ until the criterion is satisfied everywhere. The leveling $\Lambda_{h_{\mathbf{I}}}(\mathbf{I}, \mathbf{M})$ can be obtained by the following iterative algorithm:

$$
\Lambda_{h_{\mathbf{I}}}=\Lambda^{i}(\mathbf{I}, \mathbf{M})=\left[\mathbf{I} \wedge_{h_{\mathbf{I}}} \delta_{\mathbf{I}, h_{\mathbf{I}}}^{i}(\mathbf{M})\right] \vee_{h_{\mathbf{I}}} \varepsilon_{\mathbf{I}, h_{\mathbf{I}}}^{i}(\mathbf{M})
$$




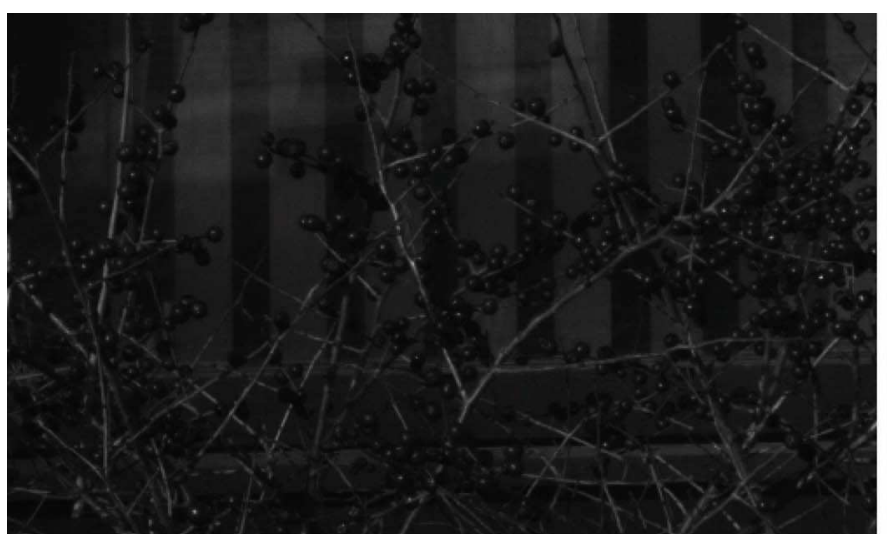

(a)

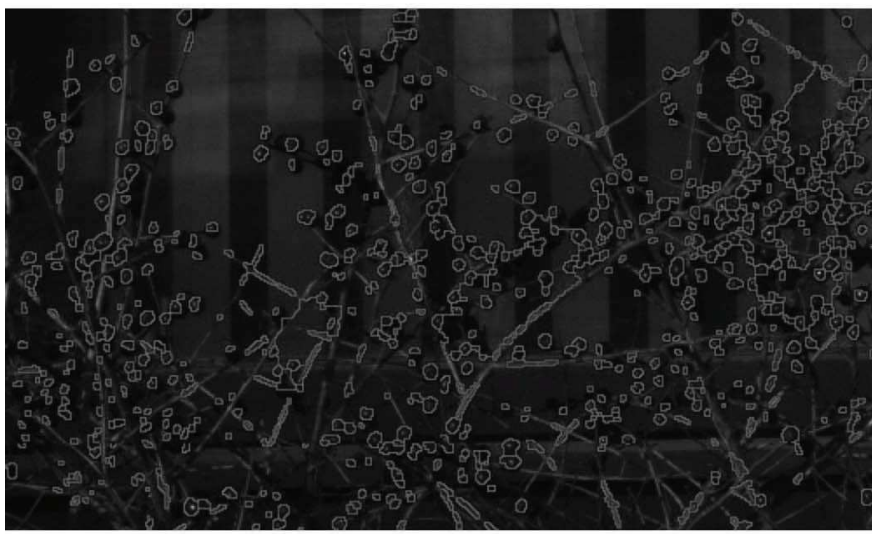

(c)

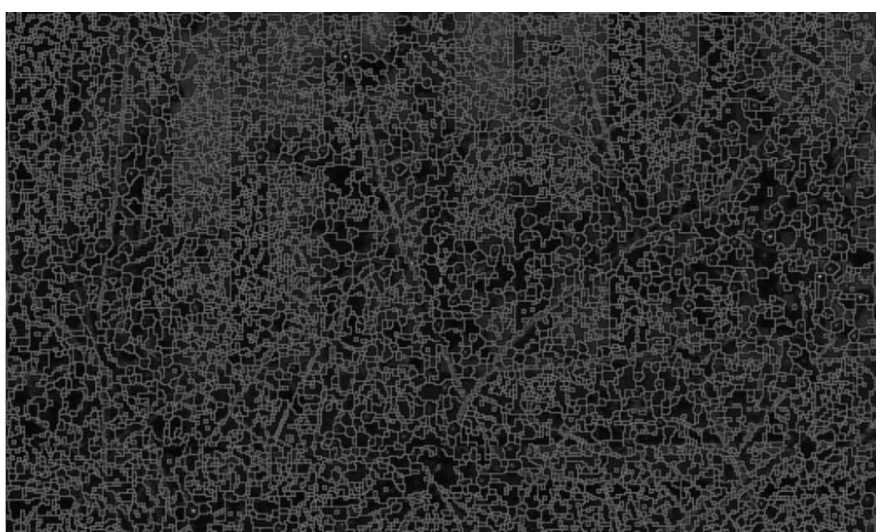

(b)

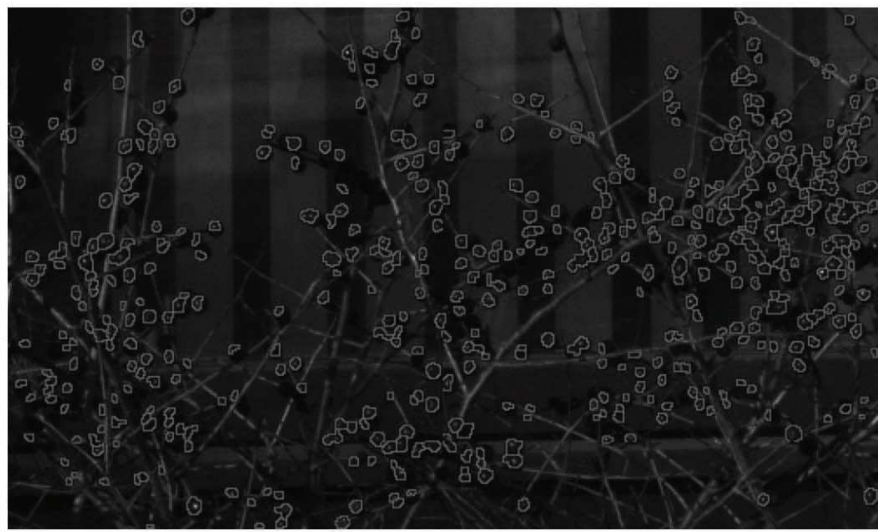

(d)

Fig. 7. Original multispectral images is the size $200 \times 500 \times 31$. Segmentation for watershed transformation with different parameters of dynamics minima of $h$-depth gradient. (a) False color image. (b) WS(I, .1); (c) WS(I, .3); (d) WS(I, .5).

such that $\Lambda^{i}(\mathbf{I}, \mathbf{M})=\Lambda_{h_{\mathbf{I}}}^{i+1}(\mathbf{I}, \mathbf{M})$ (convergence until idempotency), where the geodesic dilation (erosion) of size $i$ denotes $\delta_{\mathbf{I}, h_{\mathbf{I}}}^{i}(\mathbf{M})\left(\varepsilon_{\mathbf{I}, h_{\mathbf{I}}}^{i}(\mathbf{M})\right)$. Fig. 5 gives a real example of our method. The leveling is a simplified version of the original image, and it contains less transitions. Naturally, the simplification level is controlled by the marker image $\mathbf{M}$. In the example illustrated in Fig. 5, $\mathrm{M}$ is an opening followed by a closing with a disk of radius 10 pixels as structuring element (SE). Clearly, objects smaller that SE have been eliminated in M (Fig. 5(b)) and they are not recovered by $\Lambda_{h_{\mathbf{I}}}(\mathbf{I}, \mathbf{M})$. Thus, Fig. 5(c) is a simplified version of Fig. 5(a) where small structures have been removed but contours of larger components are preserved.

\section{Image Segmentation}

Multivariate image segmentation has been widely considered from different approaches [39]-[41]. Although theoretically feasible to extend many univariate segmentation techniques to their multivariate analogs, practical behavior is influenced by the multivariate nature of the image. Intraclass variation increases at the same time that the dimension in vector images, reducing class distinguishability and degrading segmentation performance. Multivariate imagery is specially sensitive to large intraclass variation since every component image is a variation contributor. Additionally, computational cost of segmentation algorithms increases while algorithmic robustness tends to decrease with increasing feature space sparseness and solution space complexity. We proposed to use the proposed random projection depth in combination with classical watershed transform [42] to yield a segmentation in multivariate images. The same idea can be applied to a larger family of optimization segmentation techniques [43]. A watershed transform, denoted by $\operatorname{WS}(\mathbf{I})$ associate a catch basin to each minimum of the image $\mathbf{I}$ [42]. We note in passing that in practice one often does not apply the watershed transform to the original image, but to its (morphological) gradient [7]. Basically, we apply the watershed transformation in the gradient induced by the $h$-ordering calculated by the projection depth function (3), i.e., $\boldsymbol{\Delta}_{h}(\mathbf{I})=h_{\mathbf{I}}\left(\delta_{\mathrm{SE}, h_{\mathbf{I}}}(\mathbf{I})-\varepsilon_{\mathrm{SE}, h_{\mathbf{I}}}(\mathbf{I})\right)$, where SE is typically a unitary structuring element. We notice that the gradient image, $\boldsymbol{\Delta}_{h}(\mathbf{I})$ is scalar function and consequently standard watershed algorithm can be applied on it. However, even in the case of gray scale images, the watershed transformation without any preprocessing leads to a over-segmentation. There are two possibilities to throw out the over-segmentation. The first one involves hierarchical approaches based on merging of catchment basins or based on the selection of the most significant minima according to different criteria [44]. The second one consists in determine markers for each interest region, for instance, the dynamics or contrast based transform applied to the minima of the gradient [7]. In the framework of $h$-adjunction morphology, the dynamics-based selection of minima is able to suppress minima whose $h$-adjunction is smaller than a given threshold $t$ [7]. The dynamics-based minima transform is implemented by computing a contrast closing on the scalar image 


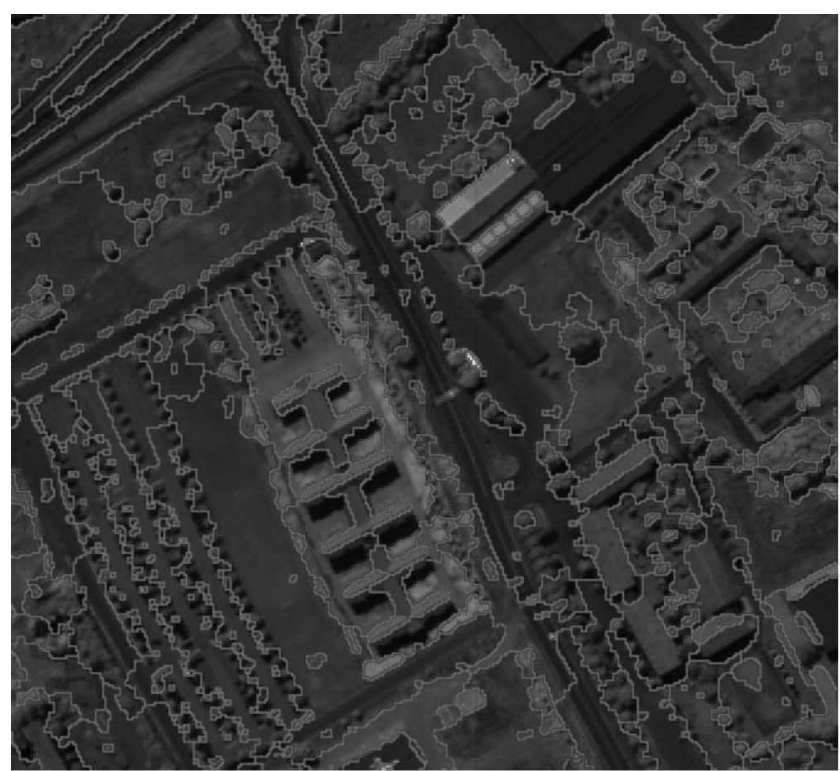

(a)

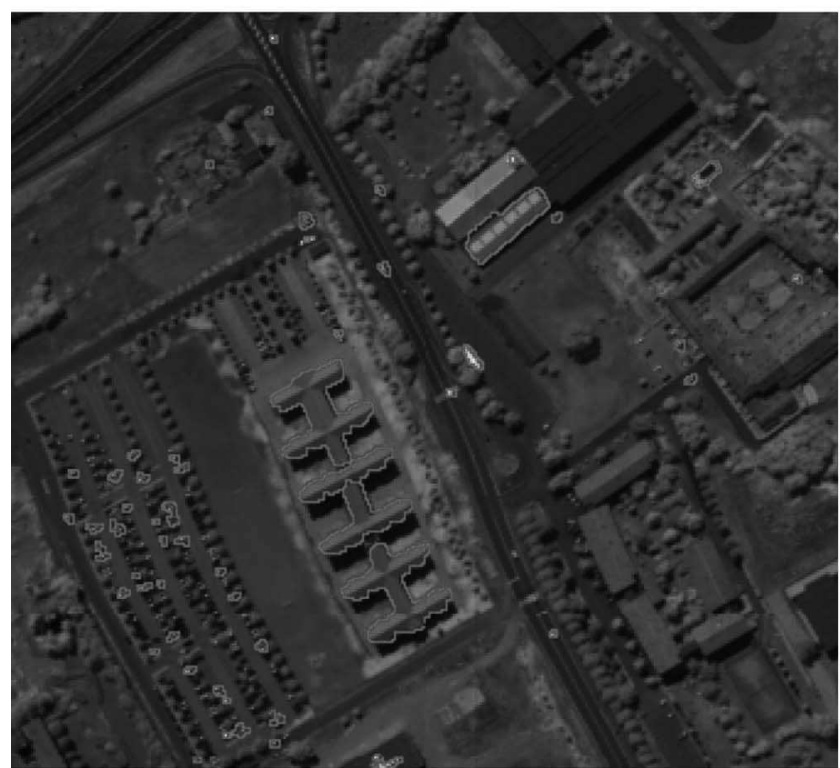

(c)

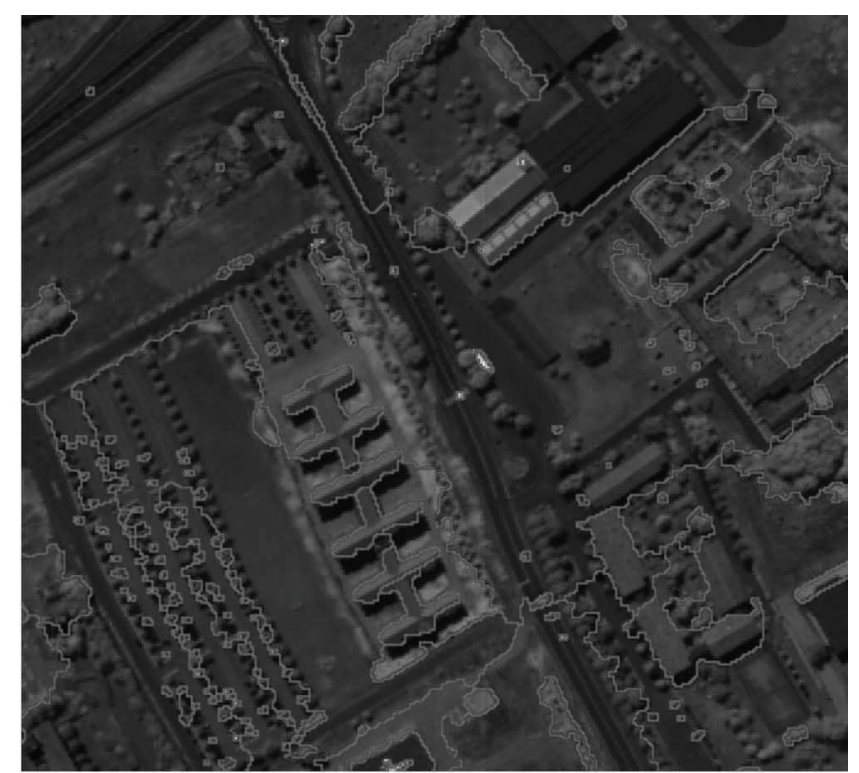

(b)

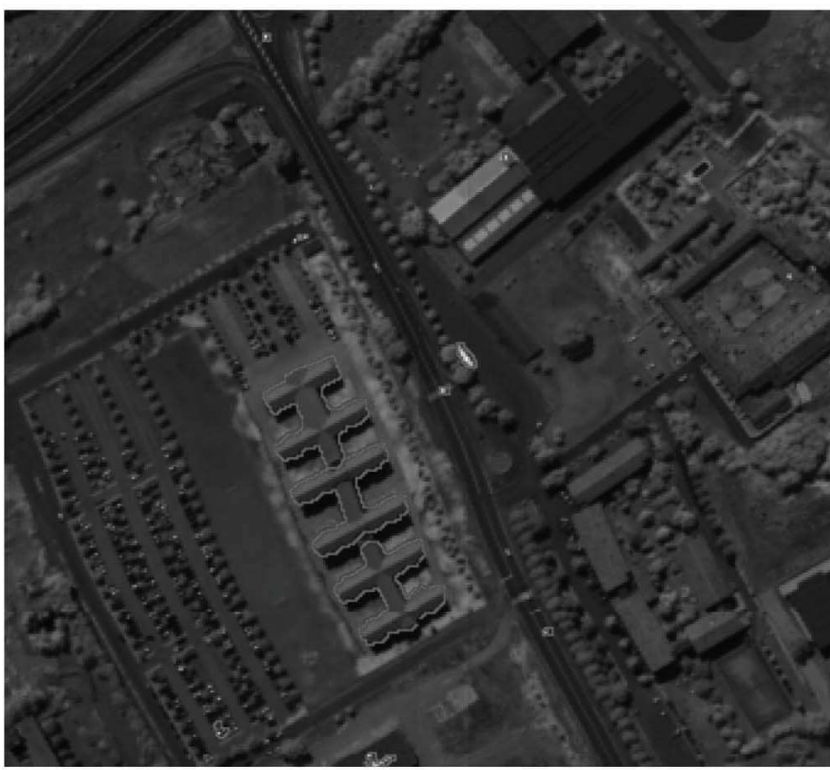

(d)

Fig. 8. Original hyperspectral image is $610 \times 340 \times 103$. Segmentation by $h$-depth watershed transformation with different parameters of dynamics minima of $h$-depth gradient. (a) False color image. (b) WS $(\mathbf{I}, .1)$; (c) WS $(\mathbf{I}, .3)$; (d) WS $(\mathbf{I}, .5)$

$\mathbf{G}$, that is a closing by reconstruction where the marker is the image after addition of the threshold $t$, i.e., $\varphi^{r e c}\left(\boldsymbol{\Delta}_{h}, \boldsymbol{\Delta}_{h}+t\right)$ [7]. We denote $\mathrm{WS}(\mathbf{I}, t)$ the watershed transform on the $h$-adjunction gradient of the image $\mathbf{I}$, where the seeds are the local minima calculated from a dynamics-based minima transform of parameter $t$ (This dynamic is normalize between 0 and 1 in the experiments). Experimental results of the segmentation strategy are shown in Figs. 6, 7 and 8. The first set of examples are color images, where the watershed segmentation produces sharp borders. An advantage of the formulation is it is directly applicable to multivariate images. Thus, we perform experiment in multispectral images from [45]. An example is the hyperspectral images Fig. 7(a), which were captured with a 31-channel camera [45]. We can observe that, our approach performs well and produce shrewd segmentation in the sense that only spectral anomalous regions are priority, for instance, fruits in Fig. 7. Fig. 8 also shows an example on a well-known hyperspectral image from the remote sensing community acquired by the ROSIS- 03 optical sensor. The original image is a 610 by 340 pixels on 103-bands. Our approach can produce a very selective foreground segmentation guided by the value of the projection depth function, i.e., buildings and some isolated pixels in the image.

\section{CONCLUSIONS}

The paper proposed the statistical depth function as a data adaptive approach to induce a vector ordering for multivariate images and consequently a framework for unsupervised multivariate mathematical morphology. Indeed, it reaches a good compromise between simplicity and effectiveness in cases where no prior information is available for a supervised approach. Multivariate segmentation based on projection depth 
function is a sort of anomaly segmentation algorithm. That is understandable because proposed method is a measure of eccentricity from a cloud point representation, where the spatial representation is not considered. The main drawback in the proposed approach is the difficulty to select the parameter $k$. It was fixed to 1000 , because in this value because we can empirically see a stability in the value of $h$-ordering for a given image. However, a theoretical study of the optimal value of $k$ will be considered in ongoing work. In the future, we are planning to further speed up the proposed ordering and sharpness enhancement algorithm, then extend the proposed method to video segmentation and enhancement.

\section{REFERENCES}

[1] S. Velasco-Forero and J. Angulo, "Mathematical morphology for vector images using statistical depth," in Mathematical Morphology and Its Applications to Image and Signal Processing, ser. Lecture Notes in Computer Science, P. Soille, M. Pesaresi, and G. Ouzounis, Eds. Berlin/Heidelberg, Germany: Springer, 2011, vol. 6671, pp. $355-366$.

[2] L. Najman and H. Talbot, Mathematical Morphology: From Theory to Applications. New York: ISTE-Wiley, June 2010.

[3] J. Goutsias and H. Heijmans, Mathematical Morphology. Amsterdam, The Netherlands: IOS Press, 2000.

[4] J. Angulo, "Morphological colour operators in totally ordered lattices based on distances: Application to image filtering, enhancement and analysis," Comput. Vis. Image Understand, vol. 107, no. 1-2, pp. 56-73, 2007.

[5] E. Aptoula and S. Lefévre, "A comparative study on multivariate mathematical morphology," Pattern Recognit., vol. 40, no. 11, pp. 2914-2929, 2007.

[6] V. Barnett, "The ordering of multivariate data (with discussion)," J. R. Statist. Soc. Ser. A, vol. 139, no. 3, pp. 318-354, 1976.

[7] P. Soille, Morphological Image Analysis. New York: SpringerVerlag, 1999.

[8] J. Serra, "The "false colour" problem," in Mathematical Morphology and Its Application to Signal and Image Processing, ser. Lecture Notes in Computer Science. Berlin/Heidelberg, Germany: Springer, 2009, vol. 5720, pp. 13-23.

[9] E. Aptoula and S. Lefévre, "On lexicographical ordering in multivariate mathematical morphology," Pattern Recognit. Lett., vol. 29, pp. 109-118, Jan. 2008.

[10] H. M. Al-Otum, "Morphological operators for color image processing based on mahalanobis distance," Opt. Eng., vol. 42, no. 9, pp. 2595-2606, 2003.

[11] S. Velasco and J. Angulo, "Hit-or-miss transform in multivariate images," in Adv. Concepts for Intell. Vis. Syst., ser. Lecture Notes in Computer Science. New York: Springer-Verlag, 2010, vol. 6474, pp. $452-462$.

[12] S. Velasco and J. Angulo, "Morphological processing of hyperspectral images using kriging-based supervised ordering," in Proc. 17th IEEE Int. Conf. Image Process. (ICIP), 2010, pp. 1409-1412.

[13] O. Lezoray, A. Elmoataz, and C. Meurie, "Mathematical morphology in any color space," in Proc. 14th Int. Conf. Image Anal. and Process. -Workshops (ICIAPW '07), 2007, pp. 183-187.

[14] A. Plaza, P. Martinez, R. Perez, and J. Plaza, "A new approach to mixed pixel classification of hyperspectral imagery based on extended morphological profiles," Pattern Recognit., vol. 37, no. 6, pp. 1097-1116, 2004.

[15] Y. Zuo and R. Serfling, "General notions of statistical depth function," Ann. Statist., vol. 28, no. 2, pp. 461-482, 2000.

[16] J. Tukey, "Mathematics and picturing data," Proc. Int. Congr. Math., pp. 523-531, 1975.

[17] R. Liu, "On a notion of data depth based on random simplices," Ann. Statist., vol. 18, pp. 405-414, 1990.

[18] D. Donoho and M. Gasko, "Breakdown properties of location estimates based on halfspace depth and projected outlyingness," Ann. Statist., vol. 20, no. 4, pp. 1803-1827, 1992.

[19] Y. Vardi and C. Zhang, "The multivariate $l_{1}$ median and associated data depth," Proc. Nat. Acad. Sci., vol. 97, no. 4, pp. 1423-1436, 2000.
[20] Y. Zuo, "Projection-based depth function and associated medians," Ann. Statist., vol. 31, no. 5, pp. 1460-1490, 2003.

[21] P. R. F. R. Hampel, E. M. Ronchetti, and W. Stahel, Robust Statist. New York: Wiley, 1986.

[22] X. Cui, L. Lin, and G. Yang, "An extended projection data depth and its application to discrimination," Commun. Statist.-Theory Meth., vol. 37 , no. 14 , pp. $2276-2290,2008$

[23] A. Cuevas, M. Febrero, and R. Fraiman, "Robust estimation and classification for functional data via projection-based depth notions," Comput. Statist., vol. 22, no. 3, pp. 481-496, 2007.

[24] Y. Zuo, "Multidimensional trimming based on projection depth," Ann. Statist., vol. 34, no. 5, pp. 2211-2251, 2006.

[25] J. Cuesta-Albertos and A. Nietos-Reyes, "The random tukey depth," Comput. Statist. Data Anal., no. 52, pp. 4979-4988, 2008.

[26] K.-T. Fang, S. Kotz, and K. W. Ng, Symmetric Multivariate and Related Distributions, ser. Monographs on statistics and applied probability. London, U.K.: Chapman \& Hall, 1990, no. 36.

[27] R. Johnson and D. Wichern, Applied Multivariate Statistics Analysis, 6th ed. London, U.K.: Prentice-Hall, 2007.

[28] J. Hoffbeck and D. Landgrebe, "Covariance matrix estimation and classification with limited training data," IEEE Trans. Pattern Anal. Mach. Intell., vol. 18, no. 7, pp. 763-767, July 1996.

[29] J. Goutsias, H. Heijmans, and K. Sivakumar, "Morphological operators for image sequences," Comput. Vis. Image Understand., vol. 62, no. 3 , pp. 326-346, 1995.

[30] S. Velasco-Forero and J. Angulo, "Supervised ordering in $\mathbb{R}^{p}:$ Application to morphological processing of hyperspectral images," IEEE Trans. Image Process., vol. 20, no. 11, pp. 3301-3308, Nov. 2011

[31] J. Serra, Image Analysis and Mathematical Morphology. Orlando, FL: Academic, 1983.

[32] J. Angulo and J. Serra, "Color segmentation by ordered mergings," in Proc. IEEE Int. Conf. Image Process., 2003, pp. 125-128.

[33] V. Caselles and J. M. Morel, "Topographic maps and local contrast changes in natural images," Int. J. Comput. Vis., vol. 33, no. 1, pp. 5-27, 1999.

[34] H. P. Kramer and J. B. Bruckner, "Iterations of a non-linear transformation for enhancement of digital images," Pattern Recognit., vol. 7, no. 1-2, pp. 53-58, 1975.

[35] P. Soille, "Constrained connectivity for hierarchical image partitioning and simplification," IEEE Trans. Pattern Anal. Mach. Intell., vol. 30, no. 7, pp. $1132-1145$, July 2008

[36] L. Pizarro, P. Mrázek, S. Didas, S. Grewenig, and J. Weickert, "Generalised nonlocal image smoothing," Int. J. Comput. Vis., vol. 90, pp. 62-87, Oct. 2010.

[37] G. Matheron, Les Nivellements, Centre de Morphologié Mathématique, 1997.

[38] F. Meyer, "The levelings," in Proc. 4th Int. Symp. Math. Morphol. and its Applicat. to Image and Signal Process. (ISMM '98). Norwell, MA: Kluwer, 1998, pp. 199-206.

[39] A. Qin and D. A. Clausi, "Multivariate image segmentation using semantic region growing with adaptive edge penalty," IEEE Trans. Image Process., vol. 19, no. 8, pp. 2157-2170, Aug. 2010.

[40] M. Grana, I. Villaverde, J. Maldonado, and C. Hernandez, "Two lattice computing approaches for the unsupervised segmentation of hyperspectral images," Neurocomputing, vol. 72, pp. 2111-2120, Jun. 2009.

[41] T. M. Nguyen and Q. J. Wu, "Dirichlet gaussian mixture model: Application to image segmentation," Image Vis. Comput., vol. 29, pp. 818-828, Nov. 2011.

[42] F. Meyer and S. Beucher, "Morphological segmentation," J. Vis. Commun. Image Represent., vol. 1, no. 1, pp. 21-46, 1990.

[43] C. Couprie, L. Grady, L. Najman, and H. Talbot, "Power watershed: A unifying graph-based optimization framework," IEEE Trans. Pattern Anal. Mach. Intell., vol. 33, no. 7, pp. 1384-1399, Jul. 2011

[44] F. Meyer, "An overview of morphological segmentation," Int. J. Pattern Recognit. Artif. Intell., vol. 15, no. 7, pp. 1089-1118, 2001.

[45] A. Chakrabarti and T. Zickler, "Statistics of real-world hyperspectral images," in Proc. IEEE Conf. Comput. Vis. Pattern Recognit. (CVPR), 2011, pp. 193-200.

Santiago Velasco-Forero, photograph and biography not available at the time of publication.

Jesús Angulo, photograph and biography not available at the time of publication. 